

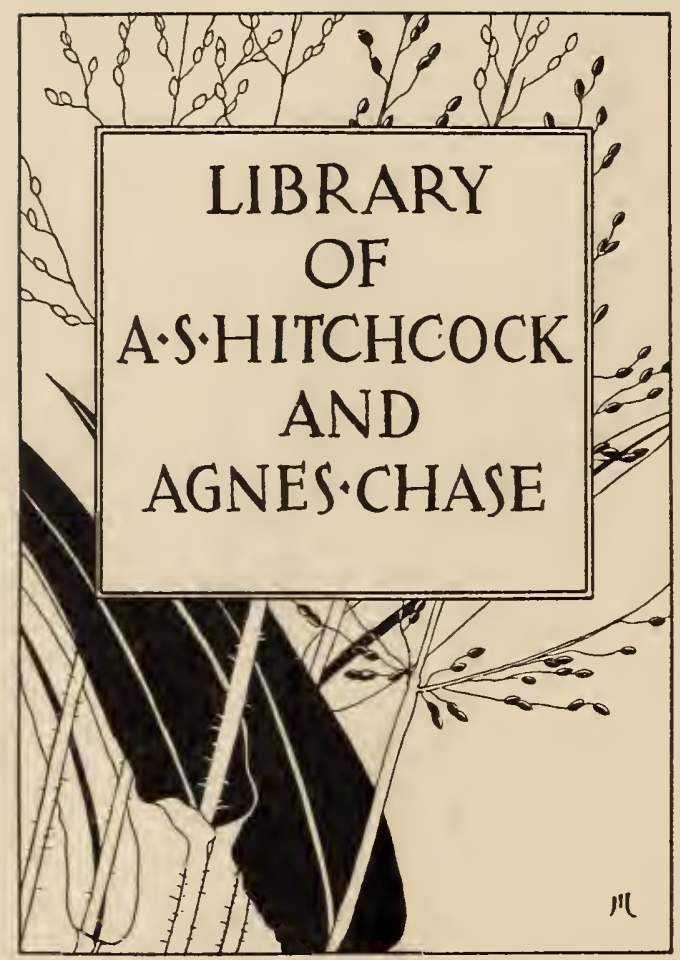




For Outline Map showing Houndary see the

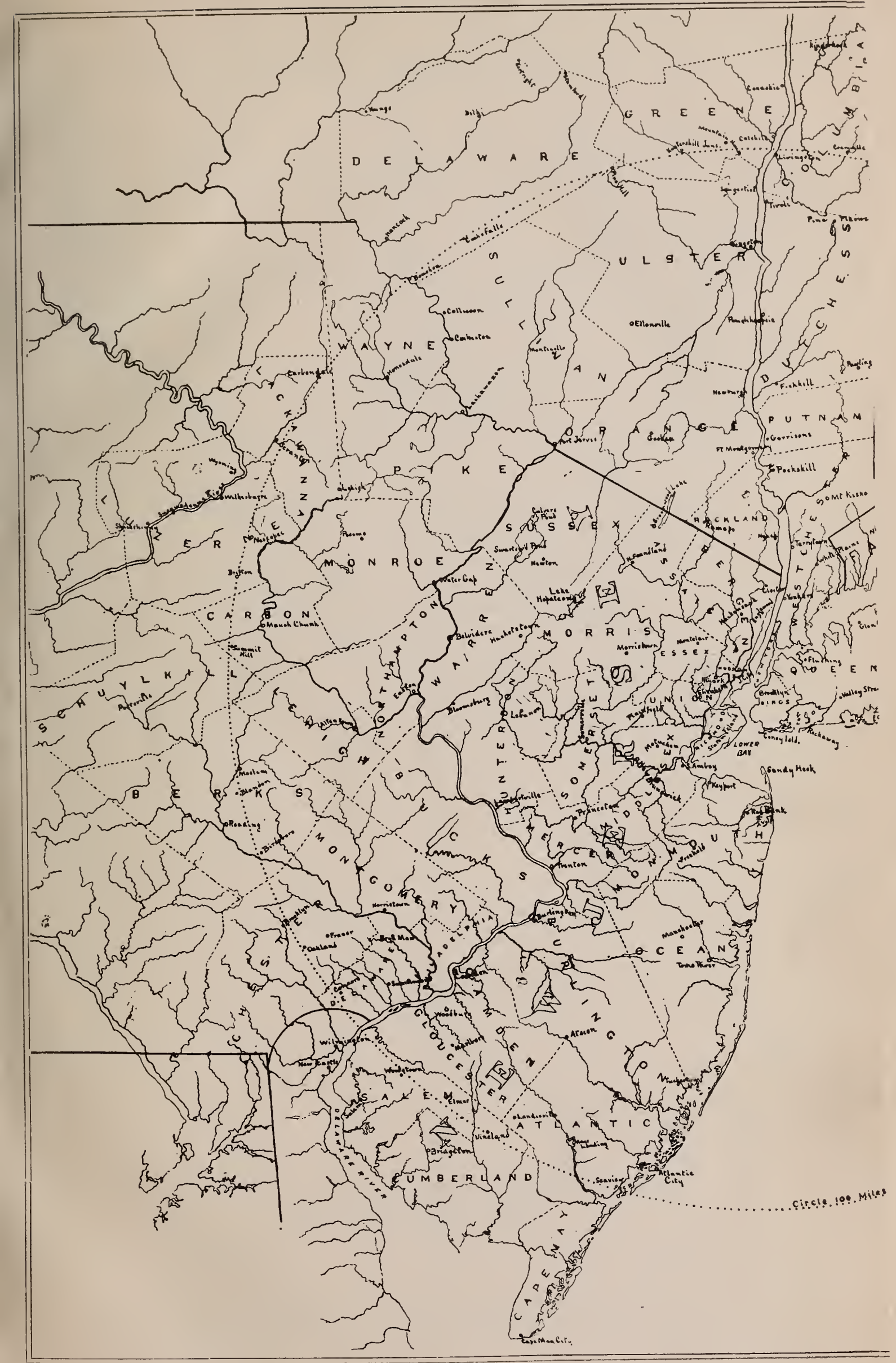



For Outline Map showlng Houndary tee the other slde.

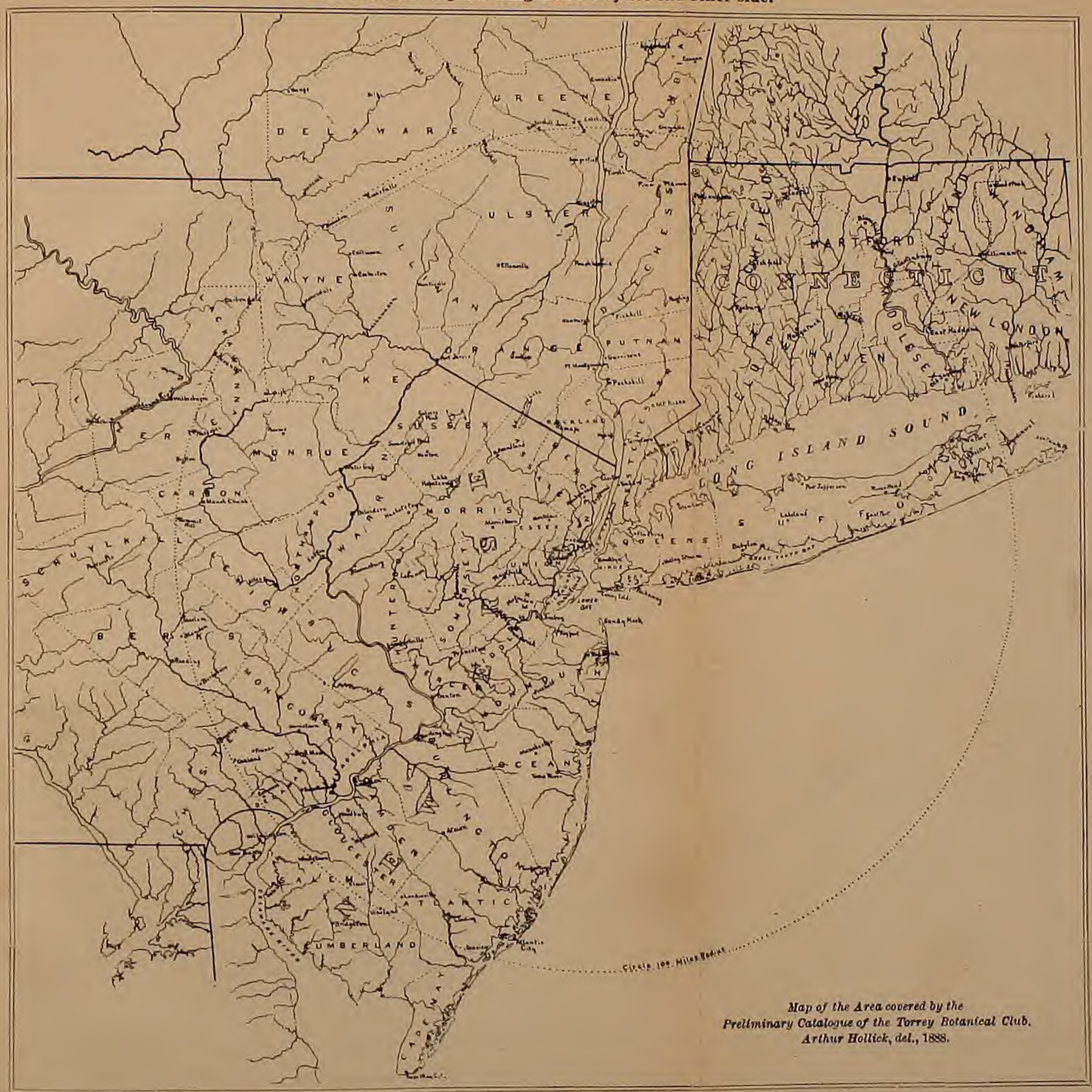




\section{otlier mide.}
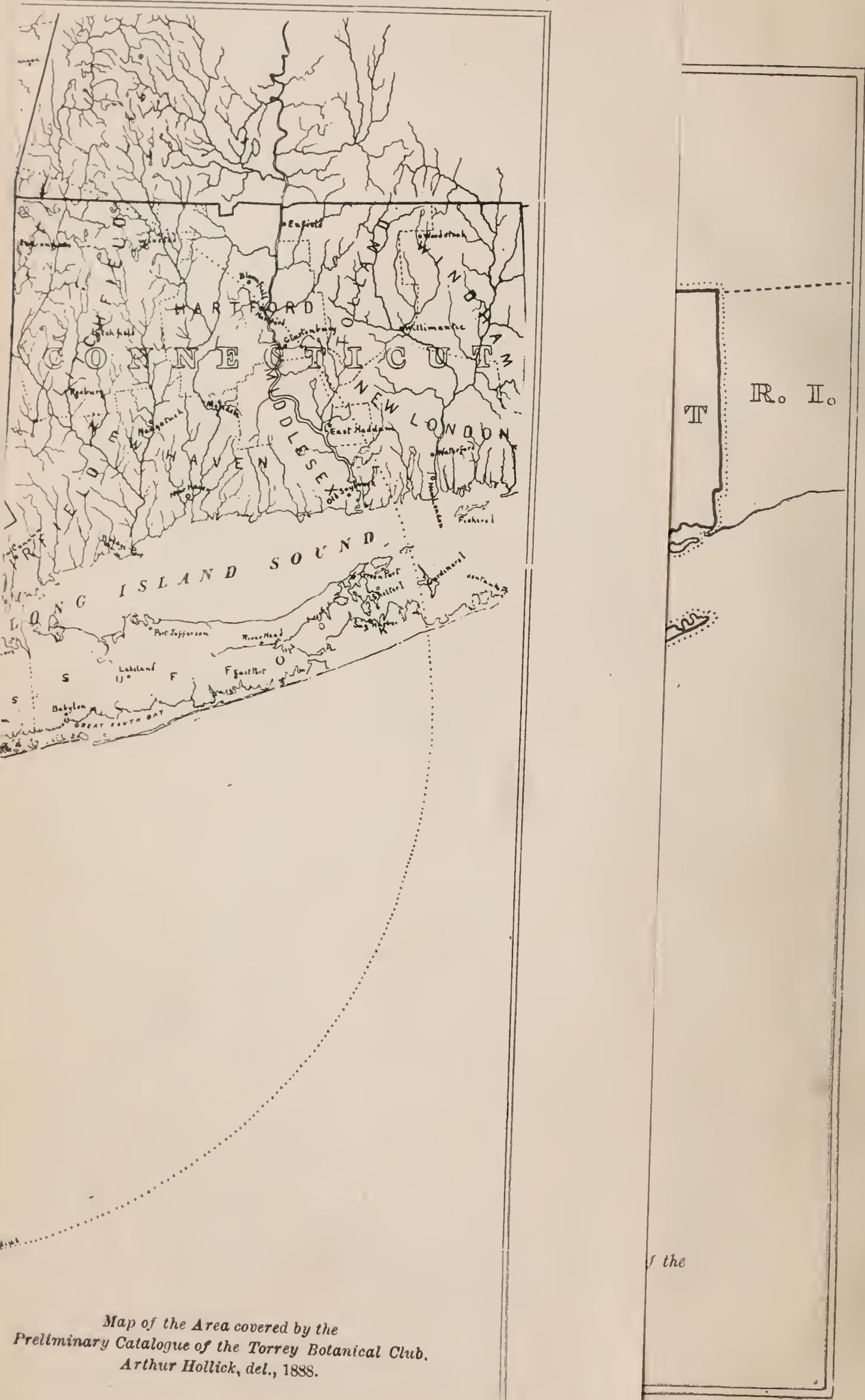


\section{A.S.HITCHCOCK.}

\section{PRELIMINARY}

\section{A T A L O G E}

OF

\section{Anthophyta and Pteridophyta}

REPORTED AS GROWING SPONTANEOUSLY WITHIN ONE HUNDRED MILES OF NEW YORK CITY.

COMPILED BY THE FOLLOWING COMMITTEE OF THE

TORREY BOTANICAL CLUB:

Justus F. POggenburg, Addison Brown,

N. L. Britton, Thos. C. Porter,

E. E. St'terns, ARTHUR HOLLICK.

THE NOMENCLATURE REVISED AND CORRECTED BY

N. L. Britton, E. E. Sterns and Justus F. Poggenburg.

New York, April 25th, I888.

Price, ONE DOLLAR.

For sale by the Secretary of the Club at the Columbia College Ierbarium. 


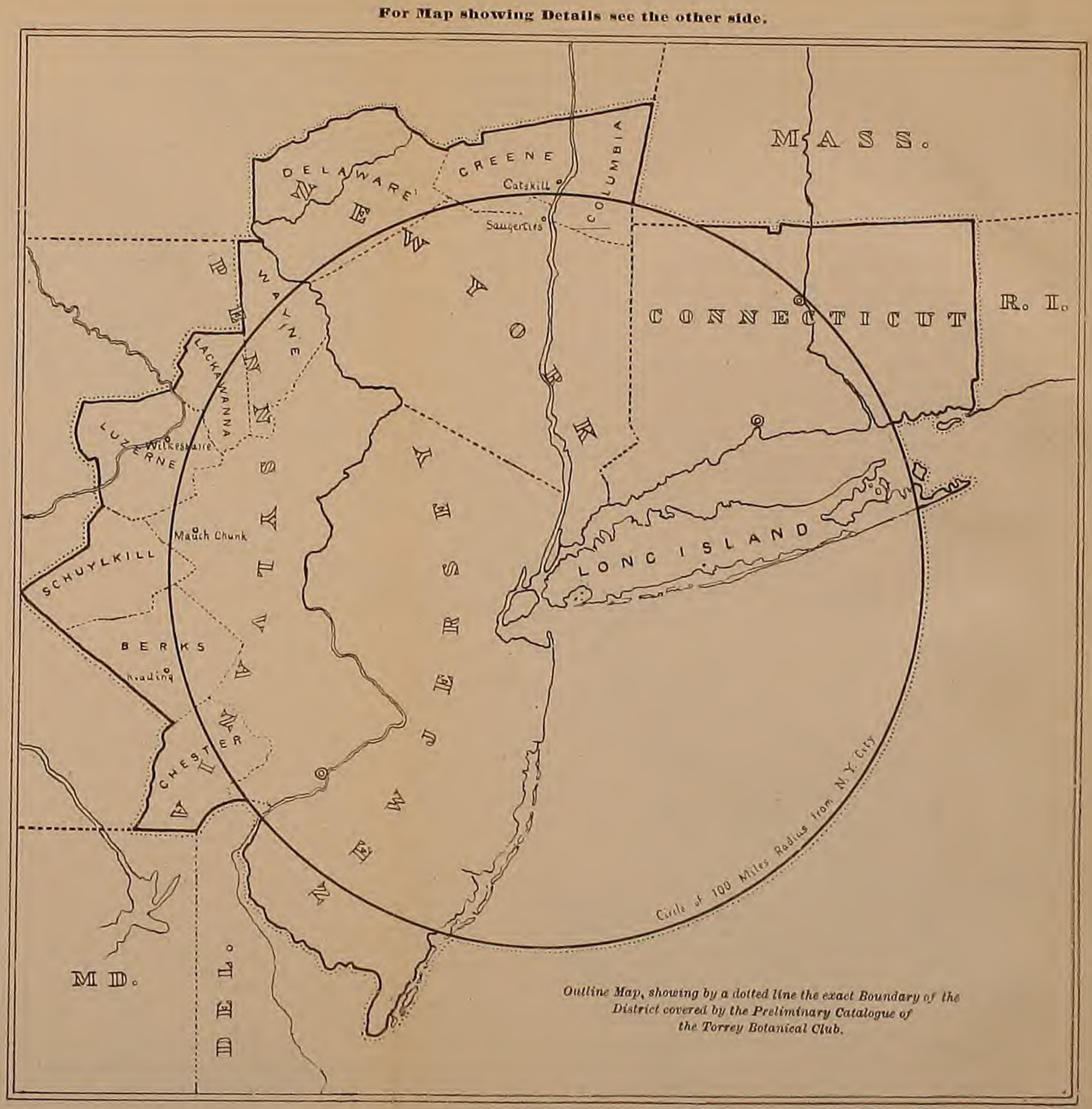






\section{CONTENTS.}

PREFACE OF THE CATALOGUE COMMITTEE, . . . . . . V V

PREFACE OF THE SUBCOMMITTEE ON NOMENCLATURE, . . viii

INDEX OF GENERA AND ORDERS, . . . . . . . . . . xi

CATALOGUE:

Polypetal. . . . . . . . . . . . . . . . . . 1

Gamopetal

Alethle, . . . . . . . . . . . . . . . . . . 44

Monocotrledones, . . . . . . . . . . . . . . . 51

Grunosierma, . . . . . . . . . . . . . . . 71

Pteridophyta. . . . . . . . . . . . . . 72

BALLAST PLANTS, . . . . . . . . . . . . . . 75 



\section{PREFACE.}

The object of this Catalogue is to furnish a compact and accessible record of the plants actually reported as growing spontaneously within one hundred miles of New York, and especially to provide a convenient check-list by means of which to secure from the botanists of the region a series of fresh and detailed reports as to the plants of their respective localities, to the end that a complete list of our Flora, with full and accurate stations for all forms of especial interest, may ultimately be prepared. Botanists engaging in this work are requested to observe the following rules:

1.-Indicate degrees of prevalence by the following terms:- "Very common," "common," "abundant," "frequent," "not rare," "rare," "very rare," "only one plant observed."

2.-Report the principal stations for all rare or local plants, indicating the locality as exactly as possible and giving the direction and distance from the nearest railway station. Precise stations for plants so rare as to be liable to be exterminated by indiscriminate collection, should, however, not be given.

3. - Note dates of commencement and end of flowering thus: April 10May 15.

4.- In all cases of doubtful identity forward specimens to the Committee for verification.

5.-Return the Catalogue when called for by the Committee with as many herbarium specimens of the plants reported as can conveniently be furnished for deposit in the Club Herbarium.

6.-Address all communications to Justus F. Poggenburg, Chairman, Committee on Local Flora, Torrey Botanical Club, Columbia College, New York City.

While the territory covered by this Catalogue is, in a general way, bounded by a circle of one hundred miles radius from the city of New York, the Committee has found it expedient to include the whole of New Jersey and Connecticut and of those counties of New York and Pennsylvania crossed by the circle. The boundary thus becomes in reality a very irregular line, but reference to the maps will at once show where it lies.

A large majority of the plants here enumerated have been personally collected by members of the Club and are represented by authentic specimens in its Herbarium. The publications which have aided in the compilation are as follows :

GENERAL LISTS.

Catalogue of Plants growing spontaneously within Thirty Miles of the City of Nero York. By John Torrey. 1819.

Bulletin of the Torrey Botanical Club. 1870-1888.

Flora of New York State. By John Torrey. 1843. 
Reports of Regents on Cabinet of Nat. Hist. of N.Y.

Manual of Botany. By Asa Gray.

Synoptical Fllora of North America. By Asa Gray.

\section{NEW YORK STATE.}

Flora of Richmond County. By Arthur Hollick and N. L. Britton. 1879.

Plantre Plandomenses, or Catalogue of Plants growing near Plandome, Long Island. By C. W. Eddy. 1807.

Catalogue of Plants growing in the Vicinity of North Salem Academy. By S. B.

Mead. 1831-2.

Report on the Fllora of Westchester County. By O. R. Willis. 1880.

Catalogue of the Phonogamnus and Acrogenous Plants growing without Cultivavation within Five Miles of Pine Plains. By Lyman Hoysradt. 1878-79.

List of Plants of Fishkill and its Vicinity. Winnifrid A. Stearns. 1880.

Catalogue of the Indigenous Plants found growing in the Vicinity of Kinderhook Academy. By W. V. S. Woodworth. 1839-40.

Plants Collected by the Botanical Class in the Delaware Literary Institute, during the S'ummer of 1840 . By M. Platt.

A Review of the Summer Birds of a Part of the Catskill Mountains, with Prefatory Remarks on the Faunal and Floral Features of the Region. By Eugene Pintard Bicknell. 1882.

Catalogus Plantarum quas sponte crescentes in insula Noveboraco observavit Johannes Leconte. 1812.

Plantce Coldenhamia, in Provincia Noveboracensi America sponte crescentes, quas ad methodus $C l$. Linncei sexualem anno 1742, etc., observavit et descripsit Cadwallader Colden. 1749-51.

\section{NEW JERSEY.}

Catalogue of Plants growing without Cultivation in the State of New Jersey, with a Specific Description of all the Violets found therein. By O. R. Willis. 1874.

Catalogue of Plants growing without Cultivation in the Counties of Monmouth and Ocean. By P. D. Knieskern, M.D. 1856.

A Preliminary Catalogue of the Fllora of New Jersey. By N. L. Britton. 1881.

\section{PENNSYLVANIA.}

An Enumeration of the Indigenous and Naturalized Plants growing in Bucks County. By I. S. Moyer, M.D. 1876.

Florula Cestrica; an Essay toward a Catalogue of the Phonogamous Plants, Native and Naturalized, growing in the Vicinity of the Borough of West Chester. By Wm. Darlington. 1826.

Flora Cestrica; an Attempt to Enumerate and Describe the Flowering and Filicoid Plants of Chester County, in the State of Pennsylvania. By Wm. Darlington. 1837. Enlarged edition, 1853.

Catalogue of the Plants of Delaware County. By Dr. Geo. Smith.

Florce Philadelphica Prodromus. By William P. C. Barton. 1815.

Compendium Florce Philadelphica, containing a Description of the Indigenous and Naturalized Plants found within a Circuit of Ten Miles around Philadelphix. By William P. C. Barton. 1818. 
List of Plants found in the Neighborhood of Philadelphia, F'ebruary to October', and List of Ferns. By Dr. Darrach. 1860.

List of the Rarer Plants found near Easton. By L. de Schweinitz. 1824.

Catalogue of Botanical Specimens collected by J. Wolle and A. L. Huebener, during the Year 1837, in the Vicinity of Bethlehem and Other Parts of Northampton County. 1839.

\section{CONNECTICUT.}

Catalogue of the Flowering Plants and Higher Cryptogams growing without Cultivation within Thirty Miles of Yale College. 1878.

List of Plants growing spontaneously in Litchfield and its Vicinity. By John P. Brace. 1822.

Catalogue of the Phonogamous Plants and the Ferns groving without Cultivation within Five Miles of Yale College. By William Tully, M.D. 1831.

Catalogue of the Phcenogamous and Vascular Cryptogamous Plants found growing in Meriden, Conn. (Incomplete.) By Emily J. Leonard. 1885.

Additional Plants found growing in Meriden, Conn., since Issue of Catalogue in 1885. By Mrs. E. B. Kendrick. 1886.

Several private herbaria and a considerable number of manuscript reports have also been carefully examined.

The work which has specially served as the basis of the present list is the Catalogue of Plants found within Thirty Miles of New York City, compiled by Mr. Wm. H. Leggett and published by him in the Bulletin (vols. i-v, 18701876), but reaching only the Gramineæ.

The preliminary labor of bringing these various reports into available form was entirely accomplished by the Chairman of the Committee, and the other members take this opportunity of recording the fact that but for his efforts this Catalogue would not have been prepared.

In the names and arrangement of orders and genera Bentham and Hooker have been closely followed, except that a few genera not accepted by them have been maintained, and the Gymnospermæ have been placed immediately before the Pteridophyta. Their convenient plan of citing Linnæus as author of the earlier generic names accepted by him has also been adopted, though reluctantly, as it is the firm conviction of the Committee that the just and proper rule would be to give full credit in all such cases to the original authors. As to specific names some important innovations have been made, as fully stated in the report of the subcommittee on nomenclature, who, alone, are responsible for all changes.

As the district includes two important seaports, New York and Philadelphia, the number of ballast plants reported is very large. These are given separately at the end, none being admitted into the Catalogue proper unless positively reported from some station other than a ballast ground.

With regard to future Catalogues the present Committee would strongly recommend that they should be descriptive and illustrated, the descriptions to be drawn from specimens collected within the district, thus making a real local Flora, of which much need is constantly felt. It would further urge that special attention be paid to the collection of the lower forms of plant life. Beyond the published lists of C. F. Austin and A. Halsey, very little information regarding the Bryophyta or Lichens is available; except a few notes by 
W. R. Gerard, E. Howe and C. H. Peck, and the descriptions of new species and list of New Jersey forms by J. B. Ellis, nothing is known of our Fungi ; and yet the determination of these plants offers no extraordinary difficulties. The recent establishment by the Club of permanent Flora Committees for both Phanerogamia and Cryptogamia affords good ground for hoping that the publication of complete descriptive lists of all the plants of this region will not be long deferred.

\section{PREFAOE OF THE SUBOOMMITTEE ON NOMENCLATURE.}

In the preparation of this Catalogue a special effort has been made to maintain for each plant as far as possible its earliest specific or varietal name. No fixed rule can be laid down for determining the generic names of plants, for the boundaries of genera are as shifting and elusive as those of states; but, as regards specific names, it is fortunately otherwise. In the case of nearly every plant it is possible to ascertain positively who first named it in accordance with the Linnæan binomial system.* The original author may have failed to refer it to the proper genus, either ignorantly or through a praiseworthy unwillingness to found new genera except on the strongest grounds. But whatever specific or varietal term may have first been applied to the plant belongs to it individually, and the most profound subsequent knowledge of it or of its relations to other plants cannot warrant any essential change in this portion of its name, always barring the cases governed by the rule that two species of the same genus must not bear the same name, or by the further rule that the generic and specific names must not be identical. Transfers from genus to genus, and alterations in rank as regards species or variety, must not (except as to gender) in any wise affect the trivial name, which is held to be absolutely fixed by the first publication, so that even the author is not at liberty thereafter to modify it in any way.

This law of priority as regards names is recognized to a greater or less extent by all botanists. Indeed, chaos would result if it were generally disregarded, and exactly in proportion as it is rigidly obeyed will order and stability be gradually evolved from the present confusion. Eminent botanists have protested, it is true, against this recognition of the early names of species when published originally in other genera, and more especially when a later name has gained acceptance. It is admitted that the first result of the strict application of this law is a considerable and undesirable increase in the number of synonyms and more or less confusion in the minds of botanists, students especially. These consequences are but temporary, however, and of no real moment weighed against the ultimate benefits to be attained.

The chief difficulty in the application of this rule is due to the fact that Linnæus himself and many of his successors have left behind them some species whose identity from lack of authentic specimens remains doubtful. Those utterly impossible of determination may be ignored altogether, but others which can be made out with some show of probability present serious

* Strictly binomial names of pre-Linnæan date are doubtless entitled to recognition. They involve, however, so many difficult questions of identity that it has been deemed best in the present work to follow the general rule and take up no names published earlier than 1753, the date of the first edition of the Species Plantarum of Linnæus. 
problems; in all such cases encountered in the preparation of this Cataloguc a conservative course has been adopted, and no name has been taken up when there was any reasonable doubt as to what plant it was meant to designate.

Another perplexing question relates to those specific names which are more or less equivalent to the accompanying generic names without being identical. Echinocystis lobata, T. \& G., was originally Sicyos echinatus, Muhl. Is Echinocystis echinata admissible? Larix Americana, Michx., was originally Pinus laricina, Du Roi. Can Larix laricina be accepted? In this Catalogue both these questions are decided afflrmatively, and those who think otherwise are referred to specularia Speculum, DC., as a weighty precedent.

Alteration of specific names has often been condoned upon the plausible pretext of making them more appropriate, and many will be disinclined to accept the strict law of priority because it brings in now and then a name which, viewed as descriptive, not ouly lacks fitness but is erroneous and misleading. The polynomial names of the pre-Linnæan systematic botanists necessarily described, and any inaccuracy was a grave defect. It is a special merit of the binomial system that descriptive terms are not essential. The personal names so often employed signify nothing as to the nature of the plants, and yet serve perfectly well to designate species. It is also true that more than one positively deceptive name is in general use. Berberis Canadensis, L., does not grow in Canada; Dioscorea villosa, L., is never villous; Salvia Hispanica, L., is "a purely Mexican species." Let these reconcile the objector to Asclepias Syriaca, L., Conioselinum Chinense, (L.), Gentiana quinquefolia, L., and some other names which are in fact erroneous, but in practice perfectly satisfactory. It is to be remembered that the same law of priority which forces these names upon us also gives us not a few that are most happily descriptive.

It may be well before concluding to explain that the author of the earliest specific or varietal name receives due credit in this Catalogue by citation in parenthesis, in all cases where his plant stands in a genus or rank other than that to which he referred it, and where consequently the name as a whole must be credited to some later authority. Ilex glabra, Gray, as commonly cited, wholly ignores Linnæus' relation to the plant; Ilex glabra, (L.), Gray, gives due and equal credit to the authors of the original specific name and the present accepted binomial.

In the list of ballast plants no attempt has been to made to apply the law of priority or to indicate the authors of transferred specific names, partly for want of time and partly because this work should be done by botanists more especially concerned with the Floras of the countries to which the plants naturally belong.

The present writers are convinced that in the not distant future the law they have taken as their guide will be generally accepted as the only one that promises a reasonable fixity of botanical names. The practice has, indeed, long been generally adopted by zoölogists, as well as by lichenologists and mycologists, and its application to flowering plants will serve to bring all biological nomenclature into practical harmony. 



\section{INI)EX OF ORIDERS ANI) GENERA.}

A

Abies ...71

Abutilon ............10, 79

Acalypha

48,88

Aoanthace a ............41

*Acanthospermum. ...82

Acer .12

Acerates .35

Achillea.... $.29,82$

Acnida .45

Aconitum............... 2

Acorus ...................57

Actra.................. 2

Actinomeris............29

*Adesmia...............79

Adiantum...............73

Adlumia................ 3

Adonis .................. 1

Ægopodium............21

Eschynomene.........13

Ethusa..................22

*Ageratuu ............. .82

Agrimonia ............. 16

Agropyrum..............71

Agrostis............68, 89

Ailanthus ...............11

Aira......................68

Ajuga..............44, 86

*Alchemilla ............ 80

Aletris ................ 53

Alisma .................58

Alismace $A$. . . . . .........58

Allium ..................54

Alnus...... .............49

Alopecurus........67, 89
*Alternanthera..........87

Althæa ............. 9, 77

Alyssum........... 4, 75

Amarantus .........44, 87

AMarantace $\&$ i ...44, 87

AMaryliIDE E.........53

Ambrosia..........28, 82

Amelanchier............17

Amianthium............55

Ammannia .............. 19

*Ammi....................81

Ammophila..............68

Amorpha ...............13

Ampelopsis...............12

AMPELIDE E..............11

Amphicarpæa .........14

Amphicarpum .........65

*Amsinckia. ............84

Anadardiade A..........12

Anacharis...............51

*Anacyclus.............82

Anagallis............34, 84

Anaphalis..............28

*Anchusa .................84

Andromeda ............33

Andropogon.......66, 89

Anemone............... 1

Anemonella............ 1

Angelica ...............22

INONAOE $\approx . . . . . . . . . . . .22$

Antennaria .............28

Anthemis ..........29, 83

Anthoxanthum ........67

Anthriscus..............21

*Anthyllis...............79
*Antirrhinum..........86

Anychia ................44

*Apera ...................89 89

A.phyllon...............40

Apios..................... 14

*Apium ..................81

Aplectrum.............51

APOCYNAOE $\mathbb{2}$............35

Apocynum ..............35

Aquilegia ............. 2

Arabis................... 4

*Arachis...... ...........80

Aralia....................22

Araliade a .............22

Arceuthobium .........47

Archangelica.............22

Archemora ..............22

Arctium..................30

Arctostaphylos ........33

Arenaria ........... 8, 84

Arethusa ................52

Argemone .............. 3

Arisæma ................57

Aristida.................67

Aristolochia ...........47

Aristolochiace \&......47

*Armeria ................884

Arnica.................. 30

*Arnoseris .... ...........83

AroIde $\alpha$.................57

Arrhenatherum ........68

Artemisia........... 30,83

Asarum.................47

AsOlepiade....... 35, 84

Asclepias................35 
Iscyrum ............. 9

Asimina................ 2

Asparagus..............53

Asperugo ...............37

*Asperula ...............81

*Asphodelus ............88

Aspidium...............73

Asplenium..............73

Asprella .................71

Aster ..............26, 82

Astragalus.........13, 79

Atriplex...........45, 87

*Atropa..................85

Avena ..............68, 89

Azalea.................... 33

Azolla................... 72

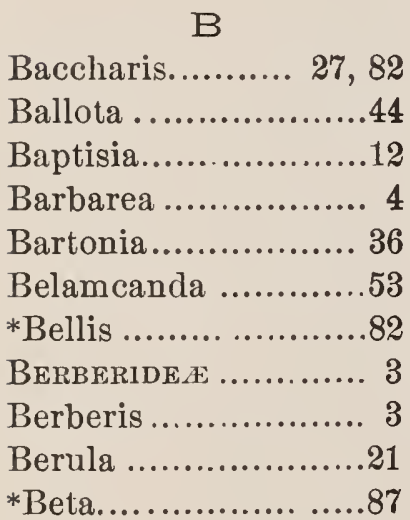

Betula..................49

Bidens ..............29, 82

*Bifora....................81 81

Bigelovia................25

Bignonia de 2 ...........41

Blephilia ................43

Blitum ...................45

Bohmeria ...............48

Bonamia................38

Borragine $\approx$........37, 84

*Borrago .................84

Botrychium ...........72

Bouteloua...............69

Brachyelytrum ........67

Brasenia................. 3

Brassica ............ 5, 75

Breweria ...............38

Briza ................69, 89

Brizopymum.............69

Bromus ............70, 90
Broussonetia.

...48

Brunella.............43, 86

Buchnera.............. 40

*Bunias ..................76

*Buphthalmum .......882

Bupleurum.........21, 81

$\mathrm{C}$
Cacalia..................30

Cacte $\ldots$...................20

Cakile .............. 5, 76

Calamagrostis...........68

Calamintha.........42, 86

*Calendula ...............83

Calla.......................57

Callitriche...............19

Calopogon...............52

Caltha.................... 2

*Calycera................81 81

*CALYOERE E .............81 81

Calystegia ...............38

Camelina................. 5

Campanula ..........32, 84

Canpanulade..... 32, 84

Camptosorus ...........73

Cannabis.................48

Capparide $\ldots$....... 5, 76

Caprifoliade $\&$....23, 81

Capsella ................. 5

Cardamine............... 4

*Cardiospermum .......78

Carduus..............30, 83

Carex...............62, 88

Carpinus .................49

*Carthamus..............83

Carum ....................21

Carya......................49

Caryophylle $\approx \ldots . .7,76$

Cassandra................33

Cassia................15, 80

Castalia .................. 3

Castanea..................50

Castilleja ................40

Catalpa ...................41

*Caucalis................8.81

Caulophyllum ......... 3

Ceanothus...............11

Celaastrine E .............11

Celastrus ................11
${ }^{*}$ Celosia...................87

Celtis...................48

Cenchrus................66

*Cenia ....................83

Centaurea..........30, 83

Cephalanthus ...........23

Cerastium.......... 8, 77

Ceratophylle \&........51

Ceratophyllum ........ 51

Cercis ......................15

Chærophyllum ....21, 81

Chamæcyparis.........71

Chamælirium...........54

Cheilanthes..............73

Chelidonium............. 3

Chelone...................39

Chenopodiatex...45, 87

Chenopodium.....45, 87

Chimaphila..............33

Chiogenes................32

Chionanthus .............35

*Chloris..................89

Chrysanthemum...29, 83

Chrysosplenium ........17

Chrysopogon ...........67

Chrysopsis..............25

Cichorium..........31, 83

Cicuta....................21

Cimicifuga ............. 2

Cinna...................68

Circæa ....................20

Cirsium................. 30

Cistine a...............6 6

Cladium.................62

*Cladothrix..............8\%

Claytonia ............ 9

Clematis................. 1

Cleome.................. 5

Clethra.....................34

Clintonia .................55

Clitoria ................... 14

Cnicus ...............30, 83

Collinsonia ..............41

*Colutea..................79

Comandra ...............47

Commelina ..............56

CommetinaCe $₫ \ldots . . . . .56$

Composit ж..........25, 82

Comptnnia ..............49 
Conifer \& ................71

Conioselinum...........22

Conium ..................21

Conobea..................39

Conoclinium.............25

Conopholis ..............40

Convallaria...............54

Convolvulaok . . 37, 85

Convolvulus ........38, 85

*Conyza ..................82

Coptis................... 2

Corallorhiza.............51

*Corchorus.............777

Corema..................51

Coreopsis...............29

*Coriandrum ...........81

Cornade................22

Cornus. ..................22

Coronilla .............13, 79

*Corrigiola ...............87

Corydalis................ 4

Corylus..................49

*Corynephorus.........89

Crantzia.................21

Crassula oex

Cratægus...............17

Crepis....................31

Crotalaria. ..............12

Croton ..............48, 88

Crotonopsis. ............48

Crudifere ......... 4, 75

Crypsis...................67

Cryptotænia...........21

Cucurbitace

Cunila ....................42

Cuphea ..................19

Cupressus................71

CUPUlifer A .............49

Cuscuta....................38

Cycloloma...............45

Cynodon .................69

Cynoglossum ............37

Cynosurus..............69

Cynthia...................31

Cyperace

Cyperus ............59, 88

Cypripedium ...........52

Cystopteris ..............73

Cytisus...................12
Dactylis.................69

Dalibarda...............15

Danthonia...............68

Datura.............38, 85

Daucus .............22, 81

Delphinium........ 2, 75

Dentaria................. 4

Deschampsia ...........68

*Desmanthus ............80

Desmodium ............13

Deyeuxia................68

Dianthera...............41

Dianthus.......... 7, 76

Diapensiade ж2...........34

Dicentra................. 3

*Dichondra ............ : 85

Dichromena............ 60

Dicksonia................74

Diervilla..................23

*Digitalis.................86

Diodia...................24

Dioscorea.................53

Dioscorea

Diospyros ................34

Diplachne..............69

Diplopappus........26, 27

Diplotaxis ......... 5, 75

Dipsace $\&$..........24, 81

Dipsacus ................24

Dirca.....................47

Discopleura ............22

Distichlis...............69

*Dorycnium ............79

Draba ................... 4

Drosera .................18

Droserace.e...........18

Dulichium...............60

\section{E}

Eatonia .................69

Ebena oe 2 .................34

*Ecbalium ...............80

Echinocystis...........20

Echinospermum...37, 84

Echium.............37, 84

Eclipta..................28

Elatine.................. 9

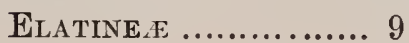

Eleocharis.........60, 61

Eleusine.......... 69, 89

Ellisia..................37

Elodea ................. .51

Elodes ................... 9

Elymus................71

*Emex ...................87

EMPetrace 2 ...........51

Epigæa ...................33

Epilobium..........19, 80

Epiphegus............. 40

EQUisetaoe $\approx \ldots . . .774 .90$

Equisetum.........74, 90

Eragrostis.........69, 89

Eranthis............... 2

Erechtites................30

Erianthus..............66

Erioadea................33

Erigeron ..........27, 82

ERiodade A. ............59

Eriocaulon............. 59

Eriophorum ...........61

Erodium ...........10, 78

*Ervum ................80

Eryngium..........21, 80

Erysimum ......... 5, 75

Erythræa................36

Erythronium ...........54

*Eschscholtzia ..........75

Euonymus..............11

Eupatorium........25, 82

Euphorbia.........47, 87

Euphorbiace.E... 47, 87

\section{F}

Fagopyrum .............46

Fagus ....................50

Fedia ...................24

Festuca.............70, 90

Ficaria.................. 1

Fidolde E..........20, 80

Filago .............28, 82

Filioes..................72

Fimbristylis.......60, 88

*Flaveria.................82

Flœrkea..................10

Fœniculum..............21

Fragaria.................16

Frangula................11 
*Frankenia..............76

*Frankeniace E ......76

Fraxinus .................35

Fuirena.

..61

Fumaria............ 4, 75

G

Galactia .................14

Galeopsis..........43, 86

Galinsoga ..............29

Galium.............24, 81

*Gaudinia................89

Gaultheria ..............33

Gaura ...............20, 80

Gaylussacia.............32

Genista .............12, 78

Gentiana .................36

Grintiane E..............36

Geran1ace æ........10, 78

Geranium ..........10, 78

Gerardia ................. 10

Geum.....................16

*Gilia.....................884

Gillenia..................15

Glaucium. .............. 3

Gleditschia ..............15

Glyceria ............70, 90

Gnaphalium........28, 82

*Gomphrena .............87

Gonolobus ...............35

Goodyera ...............52

*Gossypium ...........777

Gramine..........65, 88

Gratiola .................39

*Grindelia ...............82 82

*Guizotia................82

Gymnopogon...........69

Gymnostichum .........71

Gynandropsis...........6 6

Gypsophila....... 7, 76

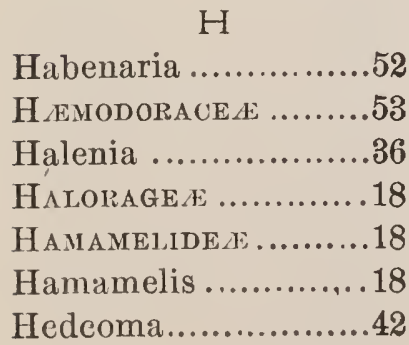

Helenium ..........29, 82

Heleocharis.............60 60

Heleochloa ............67

Helianthemum ........6 6

Helianthus .........29, 82

Heliopsis...........28, 82

Heliotropium ......37, 84

Helleborus .............. 2

Helonias .................54

Hemerocallis ............54

Hemicarpha ............61

*Hemizonia ............82

Hepatica................ 1

Heracleum .........22, 81

*Herniaria ................887

Herpestis .......... 39, 86

Hesperis ............... 5

Heteranthera ............55

*Heterotheca............82

Heuchera. ...............17

Hibiscus ................ 10

Hieracium...........31, 83

Hierochloa .........67, 89

*Hippocrepis ........... 79

Hippuris .................18

Holcus............. .68, 89

Hordeum ...........71, 90

Hottonia ..................34

Houstonia ...............24

Hudsonia .... ........... 6

Humulus................48

Hydrangea .............. 18

Hydrastis............... 2

HydrocharIDE $\&$.......5 51

Hydrocotyle ...........20

HydRopHyLLACE \& 37, 84

Hydrophyllum ......... 37

Hyoscyamus........38, 85

*Hypecoum ............75

HrPeRIOINE \&.........9, 77

Hypericum ..........9, 77

*Hypochæris...........83

Hypopitys...............34

Hypoxys ................53

Hyssopus ...............42

\section{I}

*Iberis .876

Ilex
ILICINE $A$ .11

ILLECEBRACE E . . . .44, 87

Ilysanthes. ..............39

Impatiens ...............10

Inula ...............28, 82

*Ionidium ...............76

Ipomæa............37, 85

IRIDE $\ldots \ldots \ldots \ldots \ldots . . .53,88$

Iris.................53, 88

Isanthus ...............44

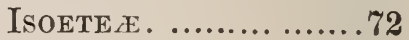

Isoetes .................. 72

Itea ...................... 18

Iva .....................28

$\mathrm{J}$

*Jasione..................84

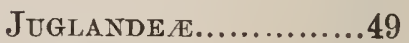

Juglans................49

JUNCACE $\mathbb{E} . . . . . . . . . . . .56$

Juncus..................56

Juniperus ..............71

Jussiæa .............19, 80

K

Kalmia ...................33

*Kochia..................87

*Kœleria ..................889

Kosteletzkya ...........10

Krigia ...................31

Kuhnia .................25

L

LABIAT $2 \ldots \ldots \ldots \ldots . . . .41,86$

Lachnanthes............53

Lactuca...................31

Lamium .................44

Laportea ................48

Lappa.................... 30

Lapsana ..................31

Larix.................... 71

Lathyrus ..........14, 80

LAURINEA...............47

Lechea ................. 6

Ledum ................. . 33

Leersia.... ..............66 66

LEGUMINOS \& ........12, 78

Leiophyllum ............33

Lemna ..................57

Lemna ...................58 
LEMNAOEA.............5\%

LENTIBUIARIE.E.........41

Leontodon ......... 31, 84

Leonurus............43, 86

Lepidium........... 5, 76

Lepigonum ............ 8

Leptochloa .........69, 89

Leptnchloa...............69

*Lepturus.................90 90

Lespedeza ...............14

Leucanthemum ..........29

Leucothoë ............... 33

Liatris...................25

Ligustrum ..............35

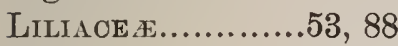

Lilium ..................54

Limnanthemum.......36

Limnobium ............51

Limosella....... ........39

Linaria.............. 39, 85

Lindera ..................47

LINE $\mathbb{E}$...............10, 77

Linnæa ...................23

Linum .............10, 77

Liparis ..................51

Lipocarpha.............61

Lippia.............41, 86

Liquidambar ...........18

Liriodendron ............ 2

Listera ..................51

Lithospermum .........37

Lobelia ...................32

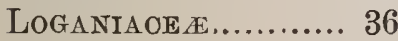

Lolium..............70, 90

Lonicera .................23

Lophanthus............43

Lophiola .................53

LORANTHAOE $1 . . . . . . . .477$

*Lotus...... ..............79

Ludwigia ................19

Lupinus............12, 78

Juzula ..................57

Lychnis............ 8, 76

Lycium ................ 38

LYCOPODIAOE E......... 72

Lycopodium ...........72

Lycopsis .................37

Lycopus ................42

Lygodium.............74
Lysimachia.............34

LYTHRARI $£ \ldots . . . . . . . .19$

Lythrum ...............19

\section{M}

Magnolia.............. 2

Magnoliacedi..........2

Maianthemum.........554

Malva .............. 9, 79

Malvaoed......... 9, 777

*Malvastrum........... .77

Marrubium ............43

Marsilia..................72

Martynia ...............41

Maruta.................29

Matricaria.........29, 83

Medeola.................55

Medicago ..........12, 78

Melampyrum ............40

*Melanthera............ 82

Melanthium ............ 55

Melastomade a........19

Melilotus...........12, 78

Melissa.................42

Menispermade A ...... 2

Menispermum......... 2

Mentha............42, 86

Menyanthes ............36

*Mercurialis.............88

Mertensia...............37

*Mesembryanthemum80

Micranthemum ........39

Microstylis .............51

Mikania ...........25, 82

Milium ................67

Mimulus.................39

Mitchella ...............24

Mitella ....................17

*Modiola.................77

*Molinia .................89

Mollugo ...........20, 80

Monarda.................43

Moneses..................33

Monotropa .............34

Monotrope \& ............34

Montelia.................45

Morus ...................48

Muhlenbergia..........67

Mulgedium ..............31
Muscari..................54

Myosotis ...........37, 84

Myrica.................49

MrRioade e..............49

Myriophyllum ..........19

\section{$\mathrm{N}$}

Nabalus...................31

NAIADACE.E .............58

Naias ..................59

*Nama ...................84

Narthecium ............54

Nasturtium........ 4, 75

Negundo ...............12

Neillia....................15

Nelumbo....... ........ 3

Nemopanthes...........11

Nepeta................. 43

Nesæa...................19

*Neslia...................76

Nicandra ................ 38

Nicotiana...........38, 85

*Nonnea..................884

Nuphar................ 3

Nymphæa.............. 3

NYMPH $\approx A O E$ \&......... 3

Nyssa...................23

Oakesia ..................55

Obolaria ................36

*Gnantlie...............81

CEnothera ...............20

Oldenlandia.............24

OLEAOE $¥$.................35

ONaGrarie E.......19, 80

Onoclea .................74

*Oncınis..................78

Onopordon ..............30

Onosmodium...........37

OPHIOGLOSSACE $E$....... 72

Ophioglossum ......... 72

Opuntia...................20

ORCHIDE \&................51

Orchis...................52

Origanum ..............42

Ornithogalum ..........54

*Ornithopus ........... 79

Оиов 
Orobanche.........40,86

Orontium . .............57

Oryzopsis..............67

Osmorhiza ..............21

Osmunda.................74

Ostrya.....................49

Oxalis....................10

Oxycoccus...............32

\section{$\mathrm{P}$}

Panicum ...........65, 89

Papaver........... 3, 75

PAPAver.ice A...... 3, 75

Pardanthus.............53

Parietaria ..........48, 88

Parnassia ...............18

*Parthenium .............82

Paspalum...........65, 89

Pastinaca...............22

*Pavonia................77

Pedalinea.........4 41, 86

Pedicularis..............40

Pellæa.....................73

Peltandra................57

Penthorum ...............18

Pentstemon .............39

*Perilla ....... ............

Petasites .................30

Petunia ............38, 85

*Peucedanum ..... .....81

Phalaris ........... 67, 89

Phaseolus ...............14

Phegopteris............73

*Philibertia....... .....84

Phleum ............68, 89

Phlox...................36

Phoradendron .........47

Phragmites.............69

Phryma..................41

Phyllanthus. .......48, 88

Physalis.............38, 85

Physostegia ............43

Phytolacca ..............46

Phytoladonoe

Picea....................71

*Picris ....................88 83

Pilea.......................48

Pimpinella .............21

Pinus
PiPelRA E A ..............47

*Pisum..................80

Plantagine........44, 86

Plantago ...........44, 86

Platanace e.............49

Platanus.................49

Pluchea .................27

Plumbagine a...... 34, 84

Poa .. ... .................70

Podophyllum........... 3

Podostemade \& .........47

Podostemon ............47

Pogonia .................52

Polanisia ........ 6, 6, 76

Polemoniace E ....36, 84

Polemonium ............36

*Polycarpon .............7 7

*Polycnemon ...........8

Polygala.................. $;$

Polygalea............. 7

Polygonace a ....46, 87

Polygonatum ..........54

Polygonum........46, 87

Polymnia ...............28

Polypodium.............72

Polypogon..............68

Polypremunı............36

Pontederia...............55

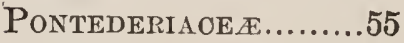

Populus ...................51

Portulaca......... 9, 77

Portula Oe. æ....... 9, 79

Potamogeton. ..........58

Potentilla..........16, 80

Poterium.................16

Prenanthes..............31

Prinuladee a...... 34, 84

Proserpinaca ............18

Prunus.....................15

Psilocarya .............60

*Psoralea.................79

Ptelea .....................11

Pteris ....................73

Pterospora ..............34

Pycnanthemum........42

Pyrola .....................33

Pyrus ....................17

Pyxidanthera............34
Quercus ................49

$\mathrm{R}$

RandNCULACE.... 1, 75

Ranunculus ....... 1, 75

Raphanus ......... 5, 76

*Rapistrum..............76

Reseda............ 6, 76

Resedace e......... 6, 76

Rhamne. ...............11

Rhamnus...............11

Rhexia...................19

Rhinanthus .............40

Rhododendron..........33

Rhodora .................33

Rhus....................12

*Rhynchosia............80

Rhyncospora ...........61 61

Rhyncospora...........60

Ribes......................18

*Ricinus.................88 88

*Richardsonia .........81

Robinia ..................13

Rosa ....................16

ROSAOEE............15, 80

Roubieva................45

Rubiadee...........23, 81

Rubus.............15, 80

Rudbeckia...............28

Ruellia ..................44

Rumex.............46, 87

Ruppia..................59

Ruta....................11

RUTAOEA................11

$\mathrm{S}$

Sabbatia ................36

Sagina .............. 8, 77

Sagittaria................58

SALIOINE \&...............50 50

Salicornia...............45

Salix .....................50

Salsola ...................46

Salvia .............43, 86

Sambucus ..........23, 81

Samolus.................. 34

Sanguinaria............ 3

Sanicula................21

SANTALAOEE............47 
SAPINDAOE.E........12, 78 Saponaria........ 7, 76

Sarracenia.............. 3

Sarraceniader.......... 3

Sassafras ...............47

Satureia............42, 86

Saururus ................47

Saxifraga................17

SAXIFliagE E ............17

*Scabiosa .................81

*Scandix .................81

Scheuchzeria ...........58

Schizæa ..................74

Schollera .................55

Schwalbea ..............40

Scirpus ...........61, 88

Scleranthus ............44

Scleria..................62

Sclerolepis .............25

*Scolymus...............83

*Scoparia ...............86

*Scorpiurus ............79

Scrophularia........39, 86

Scrophularine $2.39,85$

Scutellaria...............43

Sedum.....................18

Selaginella..............72

Selaginelle E .........7\%

Senebiera............... 5

Senecio............. 30, 83

Sericocarpus............26

*Serratula ...............83

*Sesamum...............86

*Sesbania.................79

Sesuvium...............20

Setaria...........66, 66 89

*Sherardia................81

Sicyos....................20

Sida................ 10, 77

Silene.............. 7, 76

Silphium ................28

*Silybum..................83

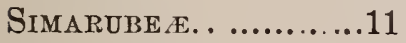

Sisymbrium..........5, 75

Sisyrinchium ...........53

Sium.....................21

Smilacina................54

Smilax ....................53

SolanaCE Æ.........38, 85
Solanum ............38, 85

Solea...................... 7

Solidago ...........25, 82

Sonchus ........... 31, 84

Sorghum .................67

Sparganium ............57

Spartina ...............66

Specularia..........32, 84

Spergula ................ 8

Spergularia............ 8

*Sphæralcea ...........77

*Spilanthes..............82

Spiræa .....................15

Spiranthes...............52

Spirodela................58

Sporobolus ........ 68, 89

Stachys........... 43, 86

Staphylea...............12

Statice ....................34

Steironema..............34

Stellaria ........... 8, 77

*Sterculiade $\nsim$.........777

Stipa......................67

Streptopus..............54

Struthiopteris ...........74

Stylosanthes ............13

Suæda.....................45

Symphoricarpos ......23

Symphytum ............37

Symplocarpus..........57

Talinum .............. 9

Tanacetum ............30

Taraxacum..............31

Taxus...................71

Tecoma..................41

*Teesdalia...............76

Tephrosia ...............13

Teucrium......... 44, 86

Thalictrum.............. 1

Thaspium ..............22

Thlaspi.................. 5

Thuja...................71

Thymeleace a.........47

Thymus ..................42

Tiarella..................17

Tiedemannia ...........22

Tilia.....................10
Tilin oefe............10, 77

Tillæa....................18

Tipularia................51

Tofieldia .................55

*Tournefortia............84

Tradescantia............56

Tragopogon ............32

*Tragus...................89

Trapa.....................20

*Trianthema ............80

*Tribulus..................78

Trichostema . ...........44

Tricuspis ................69 69

Trientalis ...............34

Trifolium ..........13, 79

Triglochin ... .........58

*Trigonella ..............78

Trillium.................55

Triodia..................69

Triosteum ................23

Triplasis.................69

Tripsacum ..............66

Trisetum ................68

Triticum..................71

Trollius ................. 2

Tsuga...................71

Tunica ................. 7

Tussilago...............30

TyPhace $2 . . . . . . . . . . . .557$

Typha...................57

U

*Ülex ...................78

Ulmus....................48

UMBELLIFE K....... 20, 80

Uniola.....................69

*Urena..................... 8 r

Urtica ...................48

URTIOACE \&..........48, 88

Utricularia ..............41

Uvularia ................55

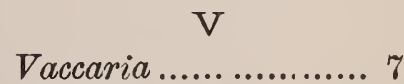

VACOINIACE . ............32

Vaccinium...............32

Valeriana ...............24

Valerianea........24, 81

Valerianella........24, 81 


\begin{tabular}{|c|c|c|}
\hline Vallisneria..............51 & VIOLARIE E........ 6, 76 & Xerophyllum . \\
\hline Veratrum ..............55 & Viti \&.....................11 & XYRIDE.E..... \\
\hline Verbascum ........ 39, 85 & & Xyris .......... \\
\hline Verbena...........44, 86 & W & \\
\hline $\begin{array}{l}\text { VerbenaOe...... 41, } 86 \\
\text { Vernomia...............25 }\end{array}$ & *Wahlenbergia .........81 & Z \\
\hline Vernonia................25 & Waldsteinia ............16 & Zannichellid. \\
\hline Veronica .............39 86 & *Waltheria ..... & Zizania....... \\
\hline Viburnum...............23 & Wolffia.......... & Zizia ............. \\
\hline Vicia..............14, 80 & Woodsia ......... & Zizia.... \\
\hline *Vigna ....................80 & Woodwardia... & Zostera...... \\
\hline Vilfa...................... & & Zygadenus........ \\
\hline Vinca .......................35 & $\mathrm{X}$ & *ZYGOPHYLLE E........ . 8 \\
\hline Viucetoxicum .........3 & Xantlium...... & *Zy gophyllum... \\
\hline Viola....................6 6 & Xanthoxylum . & \\
\hline
\end{tabular}




\section{A TALOGUE.}

\section{Clematis, $\mathbf{L}$.}

\section{RANUNCULACE $\nexists$ E.}

ochroleuca, Ait.

verticillaris, DC.

Viorna, L.

Virginiana, $\mathrm{L}$.

Thalictrum, L.

dioicum, $\mathrm{L}$.

polygamum, Muhl. (T. Cornuti, Gray, not L.)

purpurascens, L.

\section{Anemonella, Spach.}

thalictroides, (L.), Spach. (Thalictrum anemonoides, Michx.)

Anemone, $\mathbf{L}$.

acutiloba, (DC.), Lawson. (Hepatica acutiloba, DC.)

cylindrica, Gray.

dichotoma, L. (A. Pennsylvanica, L.)

Hepatica, L. (Hepatica triloba, Chaix.)

nemorosa, L.

var. quinquefolia, Gray.

Virginiana, L.

Adonis, L.

autumnalis, $L$.

\section{Ranunculus, $\mathbf{L}$.}

abortivus, $\mathbf{L}$.

var. micranthus, (Nutt.), Gray.

acris, $L$.

ambigens, S. Wats. (R. alismaefolius, Gray, not Geyer.)

aquatilis, L., var. trichophyllus, Gray.

bulbosus, $L$.

circinatus, Sibth. (R. divaricatus, Gray, Manual.)

Cymbalaria, Pursh.

fascicularis, Muhl.

Ficaria, L. (Ficaria ranunculoides, Moench.)

multifidus, Pursh.

Pennsylvanicus, L. f.

pusillus, Poir.

recurvatus, Poir. 
repens, L.

reptans, L. (R. Fllammula, I., var. reptans, Meyer.)

sceleratus, L.

septentrionalis, Poir. (R. repens, Gray, Mamml, in part.)

Caltha, L.

flabellifolia, Pursh.

palustris, $\mathrm{L}$.

Hydrastis, $\mathbf{I}$.

Canadensis, L.

Trollius, L.

Americanus, Mubl. (T'. laxus, salisb.)

Helleborus, $\mathbf{L}$.

viridis, $I$.

Eranthis, Salisb.

hyemalis, (L.), Salisl.

Coptis, Salisb.

trifolia, (L.), Salisb.

Aquilegia, L.

Canadensis, L.

var. flaviflora, (Tenney), Britton.

vulgaris, $L$.

Delphinium, $\mathbf{L}$.

Consolida, $L$.

Aconitum, $\mathbf{L}$.

Napellus, $L$.

Actax, $\mathbf{L}$.

alba, Bigel.

spicata, L., var. rubra, Ait.

Cimicifuga, L.

racemosa, (L.), Nutt.

MAGNOLIACE王.

Magnolia, $\mathbf{L}$.

glauca, L.

tripetala, L. (MI. Umbrella, Lam.)

Liriodendron, L.

Tulipifera, L.

Asimina, Adans.

ANONACE $\mathrm{E}$.

triloba, (L.), Dun.

MENISPERMACEAE.

Menispermum, $\mathbf{L}$.

Canadense, $\mathrm{L}$. 



\section{BERBERIDEAE.}

Berberis, L. vulgaris, $L$.

Caulophyllum, Michx. thalictroides, (L.), Michx.

Podophyllum, L. peltatum, L.

\section{NYMPHÆACE $\nexists$.}

Brasenia, Schreb. peltata, (Thunb.), Pursh.

Nymphra, L., Salisb. (Nuphar, Smith.) advena, Soland. (Nuphar advena, Ait.) microphylla, Pers. (Nuphar Kalmiana, Ait.) rubrodisca, (Morong), Greene. (Nuphar luteum, Gray, not Smith.)

Castalia, Salisb. (Nymphcea, L., in part.)

odorata, (Dryand), Greene.

var. minor, (Sims).

Nelumbo, Adans.

lutea, (Willd.), Pers.

Sarracenia, L. purpurea, I.

\section{Papaver, $\mathbf{L}$.}

Argemone, $L$.

dubium, $L$. somniferum, $L$.

Argemone, L.

Mexicana, $L$.

Sanguinaria, L.

Canadensis, $\mathrm{L}$.

Glaucium, Juss. luteum, Scop.

Chelidonium, $\mathbf{L}$. majus, $L$.

Dicentra, Borkh. Canadensis, (Goldie), DC. Cucullaria, (L.), DC.

\section{Adlumia, Raf.}

\section{PAPAVERACE $刃$.}

\footnotetext{
fungosa, (Ait.), Greene in litt. (A. cirrhosa, Raf.)
} 


$$
\text { , }
$$


Corydalis, DC.

aurea, (Michx.), Willd.

flavula, (Raf.), DC.

sempervirens, (L.), Pers. (C. glanca, Pursh.)

Fumaria, L.

officinalis, $L$.

\section{CRUCIFER丑.}

Nasturtium, R. Br.

Armoracia, (L.), Fries.

lacustre, Gray.

officinale, $R . B r$.

palustre, (L.), DC.

var. hispidum, (Desv.), Fisch. \& Meyer.

sylvestre, (L.), R. Bro.

Barbarea, R. Br.

precox, (Smith), R. Br.

vulgaris, $R$. Br.

var. arcuata, (Reichenh.), Loch.

Arabis, L.

Canadensis, $\mathrm{L}$.

confinis, S. Wats. (A. Drummondii, Gray, Manual.)

glabra, (L.), Bernh. (A perfoliata, Lam.)

hirsuta, (L.), Scop.

lævigata, (Muhl.), Poir.

lyrata, L.

patens, Sulliv.

\section{Cardamine, L.}

bulbosa, (Schreb.) (C.rhomlroidea, DC.)

var. purpurea, (Torr.)

diphylla, (Michx.), Wood. (Dentaria diphylla, Michx.)

heterophylla, (Nutt.), Wood. (D. heterophylla, Nutt.)

hirsuta, I.

var. sylvatica, (Link), Gray.

laciniata, (Muhl.), Wood. (D. laciniata, Mull.)

maxima, (Nutt.), Wood, (D. maxima, Nutt.)

pratensis, $\mathrm{L}$.

rotundifolia, Michx.

Alyssium, I.

calycinum, $L$.

maritimum, $L$.

Draba, L.

Caroliniana, Walt.

verna, $\mathrm{L}$. 
Hesperis, $\mathbf{I}$.

matronalis, $L$.

Sisymbrium, $\mathbf{L}$.

Alliaria, (L.), Scop.

officinale, $\left(L_{\text {. }}\right)$, Scop.

pinnatum, (Walt.), Greene. (S. canescens, Nutt.)

Sophia, $L$.

I'haliana, (L.), Gay.

Erysimum, L.

cheiranthoides, $\mathrm{L}$.

orientale, $L$.

Camelina, Crantz.

sativa, (L.), Crant:

Brassica, $\mathbf{L}$.

alba, (L.), Gray.

arvensis, (L.) (B. Sinapistrum, Boiss.)

campestris, $L$.

nigra, (L.), Koch.

Rapa, $L$.

Diplotaxis, DC.

tenuifolia, (L.), $D C$.

Capsella, Monch.

Bursa-pastoris, (L.), Mrench.

Senebiera, Poir.

Coronopus, (L.), Poir.

didyma, (L.), Pers.

Lepidium, $\mathbf{L}$.

campestre, $(L),, R . B r$.

Draba, $L$.

intermedium, Gray.

ruderale, $L$.

Virginicum, L.

Thlaspi, I.

arvense, $L$.

Cakile, Gartn.

Americana, Nutt.

Raphanus, L.

Raphanistrum, $L$.

sativus, $L$.

Cleome, $\mathbf{L}$.

CAPPARIDEAE.

pungens, Willd. 


\section{Polanisia, Raf.}

dodecandra, (Michx.) (P.graveolens, Rat.)

Gynandropsis, DC.

pertaphylla, (L.), DC.

RESEDACEAE.

Reseda, L.

Luteola, L.

Helianthemum, Pers.

CISTINE Æ.

majus, (L.) (H. Canadense, Michx.)

corymbosum, (Poir.), Michx.

Hudsonia, $\mathbf{L}$.

ericoides, L.

tomentosa, Nutt.

\section{Lechea, $\mathbf{L}$.}

Leggettii, Britton \& Hollick. (L. minor, Lam.)

var. intermedia, Britton \& Hollick.

var. pulchella, Britton \& Hollick. (L. pulchella, Rat:?)

maritima, Leggett. (I. thymifoliu, Pursh, not Michw.)

minor, L. (L. major, Michx.)

racemulosa, Lam.

tenuifolia, Michx.

thymifolia, Michx. (L. Novre-Ccesarere, Austim..)

VIOLARIE王.

Viola, I.

blanda, Willd.

rar. amœena, (LeConte). (V. amcena, LeConte.)

Canadensis, $\mathbf{L}$.

canina, L., var. Muhlenbergii, (Torr.), Gray.

lanceolata, $\mathrm{L}$.

odorata, $L$.

palmata, L.

var. cordata, (Walt.)

var. cucullata, (Ait.), Gray. (V. crcelllata, Ait.)

pedata, $\mathrm{L}$.

var. alba. Thurber.

var. bicolor, Pursh.

primulæfolia, $\mathbf{L}$.

pubescens, Ait.

var, scabriuscula, 'T. \& G. 


\section{-}


rostrata, Muhl.

rotundifolia, Michx.

sagittata, Ait.

striata, Ait.

tenella, Muhl. (V.tricolor, L., var. arvensis, DC.)

tricolor, $L$.

Solea, Ging.

concolor, (Forst.), Ging.

\section{POLYGALE正.}

\section{Polygala, $\mathbf{L}$.}

ambigua, Nutt.

brevifolia, Nutt.

cruciata, $\mathbf{L}$.

fastigiata, Nutt.

incarnata, $\mathrm{L}$.

lutea, L.

Nuttallii, T. \& G.

paucifolia, Willd.

polygama, Walt.

sanguinea, $\mathrm{L}$.

Senega, L.

verticillata, $\mathrm{L}$.

CARYOPHYLLEAE

\section{Dianthus, L.}

Armeria, $L$.

prolifer, $L$.

\section{Tunica, Scop.}

Saxifraga, (L.), Scop.

Gypsophila, $\mathbf{L}$.

arvensis, $L$. .

\section{Saponaria, L.}

officinalis, $L$.

Vaccaria, L. (Vaccaria vulgaris, Host.)

\section{Silene, $\mathbf{L}$.}

antirrtina, $L$.

Armeria, $L$.

Gallica, L., var. quinquevulnera, (J.), Kroch.

inflata, Smith.

noctiflor'a, $L$.

Pennsylvanica, Michx. 
stellata, (L.), Ait.

Virginica, L.

Lychnis, $\mathbf{I}$.

dioica, $L$.

diurna, Sil,th.

Githago, (L.), Lam.

vespertina, silith.

Cerastium, $\mathbf{L}$.

arvense, L.

var. oblongifolium, (Torr.), Hollick \& Britton.

nutans, Raf.

viscosum, $\mathrm{L}$.

vulgatum, $\mathrm{L}$.

Stellaria, L.

borealis, Bigel.

graminea, $L$.

Holostea, $L$.

longifolia, Muhl.

media (L.), Smith.

pubera, Michx.

uliginosa, Murr.

Arenaria, L.

Caroliniana, Walt. (A. squarrosa, Michx.)

Grœnlandica, (Retz.), Spreng.

lateriflora, $\mathrm{L}$.

Michauxii, (Fenzl), Hook. f. (A. stricta, MIichx.)

peploides, L.

serpyllifolia, $L$.

Sagina, $\mathbf{L}$.

apetala, L.

decumbens, (Ell.), T. \& G. (S. subulata, Groy, not Wimmer.)

var. Smithii, (Gray), S. Wats.

procumbens, $\mathrm{L}$.

Spergula, I.

arvensis, $L$.

Lepigonmm, Fries. (Sperguluria, Pers.)

medium, (L.), Fries.

var. macrocarpum, (Presl), S. Wats.

rubrum, (L.), Fries.

salinum, (Presl), Fries. 

PORTULACE王.

Portulaca, L.

grandiflora, Hook.

oleracea, $L$.

Talinum, Adans.

teretifolium, Pursh.

Claytonia, $\mathrm{L}$.

Caroliniana, Michx.

Virginica, L.

ELATINEA.

Elatine, L.

Americana, (Pursh), Arnott.

HYPERICINE无.

Ascyrum, L.

Crux-Andreæ, L.

stans, (Pers.), Michx.

Hypericum, L.

adpressum, Bart.

Ascyron, L. (H. pyramidatum, Ait.)

Canadense, L.

var. majus, Gray.

densiflorum, Pursh. (H.prolificum, L., var. densiflorum, Gray.)

ellipticum, Hook.

gentianoides, (L.) (H. Sarothra, Michx.)

gymnanthum, Engelm. \& Gray.

maculatum, Walt. (H. corymbosum, Muhl.)

mutilum, $\mathrm{L}$.

perforatum, $L$.

petiolatum, Walt. (Elodes petiolata, Pursh.)

prolificum, L.

virgatum, Lam. (H. angulosum, Michx.)

Virginicum, L. (E. Virginica, Nutt.)

\section{MALVACE正.}

Althæa, $\mathbf{L}$.

officinalis, $L$.

Malva, $\mathbf{L}$.

Alcea, $L$.

moschata, $I$.

rotundifolia, $L$.

sylvestris, $L$. 
. 
Sida, I.

spinosa, $L$.

Abutilon, Gaertn.

.4vicennce, Grert".

Kosteletzkya, Presl.

Virginica, (L.), Gray.

Hibiscus, L.

Moscheutos, L.

Syriacus, $I$.

'Trionum, $L$.

\section{TILIACEA.}

Tilia, L.

Americana, L.

pubescens, Ait. ('T. Americana, L., var. pubescens, Gray.)

LINEÆ.

Linum, L.

striatum, Walt.

sulcatum, Ridd.

usitatissimum, $L$.

Virginianum, L.

GERANIACE $\mathbb{E}$.

Geranium; I.

Carolinianum, $\mathbf{L}$.

columbinum, $L$.

dissectum, $L$.

maculatum, $\mathrm{L}$.

pusillum, $L$.

Robertianum, L.

Sibiricum, $L$.

Erodium, L'Hér.

cicutarium, (L.), L'H'r

Florkea, Willd.

proserpinacoides, Willd.

Oxalis, L.

Acetosella, L.

corniculata, L.

var. stricta, (L.), Sav.

violacea, $\mathbf{L}$.

Intpatiens, I.

aurea, Muh]. (I. pallida, Nutt.)

biflora, Walt. (I. fulva, Nutt.) 

RUTACE压.

Ruta, L.

graveolens, $L$.

Xanthoxylum, L.

Americanum, Mill.

Ptelen; I.

trifoliata, $\mathbf{L}$.

Ailanthus, Desf.

\section{SIMARUBEA.}

glandulosus, Desf.

ILICINE $\approx$.

Ilex, I.

dubia. (Don). (I. mollis, Gray.)

glabra, (L.), Gray.

lævigata, (Pursh), Gray.

monticola, Gray.

opaca, Ait.

rerticillata, (L.), Gray.

var. tenuifolia, Torr.

Nemopanthes, Raf.

Canadensis, (Michx.), DC.

CELASTRINE王.

Enonymis, $\mathbf{L}$.

Americanus, L.

var. obovatus, T. \& G.

atropurpureus, Jacq.

Europoeus, $T$.

Celastrus, L.

scandens, L.

RHAMNE 2 .

Rhammis, L.

alnifolia, L'Hér.

Caroliniana, Walt. (Frangula Caroliniana, Gray.) cathartice, $L$.

Ceanothus, I.

Americanus, L.

AMPELIDE E.

Vitis, L.

æstivalis, Michx.

cordifolia, Michx.

Labrusca, L. 
quinquefolia, (L.), Lam. (Ampelopsis quinquefolia, Michx.) riparia, Michx. (V. cordifolia, Michx., var. riparia. Gray.)

\section{SAPINDACEF.}

Acer, L.

dasycarpum, Ehrh.

Pennsylvanicum, L.

rubrum, L.

saccharinum, Wang.

var. nigrum, (Michx. f.), T. \& G.

spicatum, Lam.

Negundo, Monch.

aceroides, Mœnch.

Staphylea, I.

trifolia, L.

ANACARDIACE正.

Rhus, I.

aromatica, Ait.

copallina, L.

glabra, L.

Toxicodendron, L.

var. radicans, (L.), Torr.

typhina, L.

venenata, DC.

LEGUMINOS正.

Baptisia, Vent.

tinctoria, (L.), R. Br.

Crotalaria, I.

sagittalis, $\mathrm{L}$.

Lupinus, $\mathbf{I}$.

perennis, $\mathrm{L}$.

Genista, $\mathbf{I}$.

tinctoria, L. (Near Peekskill?)

Cytisus, L.

scoparius, Link.

triflorus, L'Hér.

Merlicago, L.

lupulina, $I$.

maculata, Willd.

sativa, $I$.

Mrlilotus, Juss.

alba, Lam. 
officinalis, (L.), Lam.

Trifolinm, L.

agrarium, $L$.

arvense, $L$.

Carolinianum, Michx.

hybridum, $L$.

incarnatum, $L$.

medium, $L$.

pratense, $I$.

procumbens, $L$.

var. minus, Koch.

reflexum, L.

repens, $L$.

Amorpha, L.

fruticosa, $L$.

Tephrosia, Pers.

Virginiana, (L.), Pers.

Robinia, I.

hispida, $L$.

Pseudacacia, $L$.

viscosa, Vent.

Astragalus, L.

Canadensis, L. (Westchester County?)

Coronilla, L.

varia, $L$.

Eschynomene, L.

Virginica, (L.) (AE. hispicla, Willd.)

Stylosanthes, Swartz.

biflora, (L.) (S. elatior, Swartz.)

Desmodium, Desv.

Canadense, (L.), DC.

canescens, (L.), DC.

ciliare, (Muhl.), DC.

cuspidatum, (Muhl.), Hook.

Dillenii, Darl.

grandiflorum, (Walt.), DC. (D. acuminatum, $D C$.)

humifusum, (Muhl.), Beck.

lævigatum, (Nutt.), DC.

Marylandicum, (L.), Boott.

nudiflorum, (L.), DC.

paniculatum, (L.), DC.

pauciflorum, (Nutt.), DC. 
rigidum, (Ell.), DC.

rotundifolium, (Michx.), DC.

strictum, (Pursh), DC.

viridiflorum, (L.), Beck.

\section{Lespedeza, Michx.}

angustifolia, (Pursh), Eil. (I. capitata, Michx., var. angustifolia, Pursh.)

frutescens, (Willd.), Ell. (L. capitate, Michx.)

hirta, (L.), Ell.

var. angustifolia, Maxim.

repens, (L.), Bart.

reticulata, (Muhl.), Pers. (L. violacea, Pers., var. sessiliflora, Don.)

var. angustifolia, (Hook.), Maxim. (L. violacea, Pers., var. aingustifolia, T. \& G.)

Stuvei, Nutt.

violacea, (L.), Pers.

Vicia, $\mathbf{L}$.

Americana, Muhl.

Caroliniana, Walt.

Cracca, L.

hirsuta, (L.), Koch.

sativa, $L$.

var. angustifolia, (Roth), Ser.

tetrasperma, $(L$.$) , Lois.$

\section{Lathyrus, I.}

giaucifolius, Beck. (L. ochroleucrus, Hook:)

maritimus, (L.), Bigel.

palustris, $\mathrm{L}$.

var. myrtifolius, (Muhl.), Gray.

venosus, Muhl.

\section{Clitoria, I.}

Mariana, L.

\section{Amphicarpaea, Ell.}

comosa, (L.), Ridd. (A. monoica, Ell.)

Apios, Monch.

tuberosa, Mœnch.

\section{Galactia, P. Br.}

pilosa, Ell. (G. mollis, Gray, not Michx.)

regularis, (L.) (G. glabella, Michx.)

\section{Phaseolus, I.}

angulosus, (Muhl.), Ort. (P. diversifolius, Pers.) 
helvolus, L.

polystachyus, (L.) (I.perennis, Walt.)

Gleditschia, L.

triacanthos, $L$.

Cassia, L.

Chamæcrista, L.

Marylandica, L.

nictitans, $\mathrm{L}$.

Cercis, $\mathbf{L}$.

Canadensis, L.

Prunus, L.

ROSACEA.

Americana, Marsh.

Cerasus, $L$.

Mahaleb, $L$.

maritima, Wang.

var. pygmæa, (Willd.)

Pennsylvanica, L. f.

pumila, L.

serotina, Ehrh.

spinosa, $L$.

Virginiana, L.

Spiraen, L.

Aruncus, L.

betulifolia, Pall. (S. corymbosa, Raf.)

lobata, Jacq.

salicifolia, L.

tomentosa, L.

Neillia, Don.

opulifolia, (L.), Benth. \& Hook. (Spircea opulifolia, L.)

Gillenia, Møench.

trifoliata, (L.), Mœench.

Rubus, L.

Canadensis. L.

cuneifolius, Pursh.

Dalibarda, L. (Dalibarda repens, I.)

hispidus, $\mathrm{L}$.

laciniatus, Willd.

neglectus, Peck.

occidentalis, L.

odoratus, L.

strigosus, Michx. 
triflorus, Richards.

villosus, Ait.

var. frondosus, Torr.

var. humifusus, T. \& G.

Geum, I.

album, Gmel.

var. flavum, Porter.

rivale, $\mathrm{L}$.

strictum, Ait.

vernum, (Raf.), T.\& $G$.

Virginianum, L.

Waldsteinia, Willd.

fragarioides, (Michx.), Tratt.

Fragaria, L.

Indica, Andr.

vesca, L.

Virginiana, Duchesne.

var. Illinoensis, (Prince), Gray.

\section{Potentilla, L.}

anserina, $\mathrm{L}$.

argentea, $\mathrm{L}$.

arguta, Pursh.

Canadensis, L.

var. simplex, (Michx.), T. \&. F.

fruticosa, L.

Norregica, L.

palustris, (L.), Scop.

recta, $L$.

tridentata, Soland.

Agrimonia, L.

Eupatoria, L.

parviflora, Ait.

Poterium, I.

Canadense, (L.), Gray.

Sanguisorba, $L$.

\section{Rosa, $\mathbf{L}$.}

blanda, Ait.

canina, $L$.

Carolina, L.

humilis, Marsh. (R. lucida, Gray, Mamual, in part.)

var. lucida, (Ehrh.), Best. (R. hucida, Ehrh., in part.)

var. villosa, Best. (R. lucida, Gray, Manual, in part.) 
nitida, Willd. (R. Incida, Grery, Manuru, in part.)

mbiginosa, I. (Includes R. microntha, Smith.)

setigera, Michir.

Pyrus. I.

Americana, (Marsh.), DC.

angustifolia, Ait.

arbutifolia, (L.), L.f. ( P. arbutifolia, L., var. erythrocarya, Gray)

var. melanocarpa, (Willd.), Hook.

coronaria, L.

Malus, L.

Cratagus, I.

coccinea, L.

var. macrantha, Dudley.

Crus-galli, L.

O.xyacantha, I.

parvifolia, Ait.

populifolia, Walt. (C. cordata, Ait.)

Pyracantha, (L.), Pers.

spathulata, Michx. (Near Philadelphia?)

subvillosa, Schrad.

tomentosa, L.

var. punctata, (Jacq.), Gray.

\section{Amelanchier, Lindl.}

Canadensis, (L.), Medikus (fide Steudel.)

var. obovalis, (Michx.) (var. oblongifolit, T. \& Gr.)

var. oligocarpa, (Michx., T. \& G.

var. rotundifolia, (Michx.), T. \& G.

\section{SAXIFRAGE正.}

Saxifiraga, L.

micranthifolia, (Haw.) (S. erosc, Pursh.)

Pennsylvanica, L.

Virginiensis, Michx.

var. pentadecandra, Sterns.

Tiarella, L.

cordifolia, L.

Mitella, I.

diphylla, L.

nuda, L.

Heuchera, I.

Americana, L.

Chrysosplenium, I. 
Americanum, L.

Paruassia, I.

Caroliniana, Michx.

Hydrangea, $\mathrm{I}$.

arborescens, L.

Itea, $\mathbf{I}$.

Virginica, L.

Ribes, $\mathbf{I}$.

Cynosbati, L.

floridum, L'Hér.

lacustre, Poir.

oxyacanthoides, L. (R. hirtellum, Michx.)

prostratum, L'Hér.

rotundifolium, Michx.

rubrum, $L$.

CRASSULACE王.

Tillaea, L.

simplex, Nutt.

Sedum, L.

acre, $L$.

roseum, (L.), Scop. (S. Rhodiola, DC.)

Telephium, L.

ternatum, (Haw.), Michx.

Penthorum, I.

sedoides, $\mathrm{L}$.

DROSERACEA.

Drosera, I.

filiformis, Raf.

intermedia, Drev. \& Hayne, var. Americana, DC. (D. longifolia, Michx., not $L$.)

rotundifolia, $\mathrm{L}$.

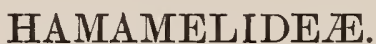

\section{Hamamelis, $\mathbf{L}$.}

Virginiaua, L.

Liquidambar, $\mathrm{L}$.

styraciflua, L.

HALORAGE

Proserpinaca, $\mathbf{L}$.

palustris, L.

pectinata, Lam.

Hippuris, L.

vulgaris, $\mathrm{L}$. 
Myriophyllum, L.

ambiguum, Nutt. (M. ambigunem, Nutt., var. natans, 'T' \& Gr.) var. capillaceum, T. \& G.

var. humile, (Raf.) (M. arnbiguum, Nutt., var. limosum, 'Torr.) heterophyllum, Michx.

pinnatum, (Walt.) M. scabratum, Michx.)

spicatum, L.

tenellum, Bigel.

verticillatum, $\mathrm{L}$.

Callitriche, I.

Austini, Engelm.

heterophylla, Pursh.

var. linearis, (Pursh), Austin.

verna, L.

MELASTOMACE无.

Rhexia, I.

Mariana, L.

Virginica, L.

LYTHRARIEAE.

Ammannia, L. coccinea, Rottb.

latifolia, L.

ramosior, L. (A. humilis, Michx.)

Cuphea, P. Br.

petiolata, (L.), Kœhne. (C. viscosissima, Jacq.)

Lythrum, L.

alatum, Pursh.

hyssopifolia, L.

lineare, L.

Salicaria, $L$.

Nesaea, Commers.

verticillata, (L.), HBK.

Epilobium, L.

ONAGRARIE正.

coloratum, Muhl.

palustre, L., var. oliganthum, (Michx.) (var. lineare, Gray.)

spicatum, Lam. (E. angustifolium, Gray, Manual.)

strictum, Mubl. (E. molle, 'Torr.)

Jussiaen,' $\mathbf{T}$.

repens, $T$.

Ludwigia, L. 


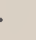


alternifolia, L.

hirtella, Raf.

linearis, Walter.

palustris, (L.), Ell.

polycarpa, Short \& Peter.

sphærocarpa, Ell.

Enothera, I.

biennis, $\mathrm{L}$.

var. grandiflora, (Ait.), Lind].

var. muricata, (L.), Lindl.

fruticosa, L.

var. hirsuta, Nutt.

var. humifusa, Allen. (OE. linearis, Gray, in part.)

var. linearis, (Michx.), S. Wats. (OE. linearis, Gray, in part, and OE. riparia, Nutt.)

humifusa, Nutt.

pumila, L. (UE. chrysantha, Michx.)

sinuata, L.

var. minima, (Pursh), Nutt.

\section{Gaura, I.}

biennis, L.

Circrea, I.

alpina, $\mathrm{L}$.

Lutetiana, L.

Trapa, I.

natans, $L$.

CUCURBITACE\#.

Echinocystis, T. \& G.

echinata, (Muh].) (E. lobata, T. \& G.)

Sicyos, $\mathbf{L}$.

angulatus, $I$.

Opuntia, Mill.

CACTEA.

Rafinesquii, Engelm.

vulgaris, Haw.

Sesuvium, L.

FICOIDEસ.

maritimum, (Walt.) (S. Portulacastrum, Gray, not L.)

Mollugo, L.

v'erticillata, $I$.

Hydrocotyle, $\mathbf{L}$.

UMBELLIFERÆ.

Americana, L. 
ambigua, (Gray.) (H. umbellatı, L., var. combifuce. Grouy.) interrupta, Muh].

ranunculoides, L. f.

umbellata, $\mathrm{L}$.

Eryngium, 1 .

Virginianum, Lam.

yuccrefolium, Michx.

Sanicula, L.

Canadensis, L.

Marylandica, L.

Conium, I.

maculcutım, $L$.

Bupleurum, I.

rotundifolium, $L$.

Cicuta, $\mathbf{L}$.

bulbifera, L.

maculata, L.

Carum, L.

Carri, $L$.

Sium, L.

Carsonii, Durand.

cicutæfolium, Gmel. (S. lineare, Michx.)

Berula, Koch.

angustifolia, (L.), Koch. (Sium angustifolium, L.)

Egopodium, L.

Podagraria, $L$.

Pimpinella, $\mathbf{L}$.

integerrima, (L.), Benth. \& Hook. (Zizia integerrima. DC.)

Saxifraga, L., var. major, Koch.

Cryptotænia, DC.

Canadensis, (L.), DC.

Osmorhiza, Raf.

Claytoni, (Michx.) (O. brevistylis, $I C^{Y}$.)

longistylis, (Torr.); DC.

Chærophyllum, L.

procumbens, (L.), Crantz.

Anthriscus, Hoffim.

Cerefolium, (L.), IIoffm. (Chcerophyllum sutivum, Lam.)

Foeniculum, Adans.

vulgare, Gerertn.

Crantzia, Nutt.

lineata, (Michx.), Nutt. 

Discopleura, DC.

major, (Walt.) (D. capillacea, DC.)

Ethusa, L.

Cynapium, $L$.

Thaspium, I.

aureum, (L.), Nutt.

var. cordatum, (Walt.) (T. trifoliatum, Gray.)

var. trifoliatum, (L.) (var. atropurpureum, Gray.)

barbinode, (Michx.), Nutt.

Zizia, Koch.

aurea, Koch. (Thaspium aureum, var. apterum, Gray.)

cordata, Koch. (Thaspium trifoliatum, var. apterum, Gray.)

Conioselinum, Fisch.

Chinense, (L.) (C. Canadense, T. \& G.)

Angelica, L.

atropurpurea, I. (Archangelica atropurpurea, Hoffm.)

villosa, (Walt.) (Archangelica hirsuta, 'T.\& G.)

Pastinaca, $\mathbf{L}$.

sativa, $L$.

Tiedemannia, DC.

rigida, (L.), Coulter \& Rose. (Archemora rigida, $D C$.)

var. longifolia, (Pursh.) (A. rigida, var. ambigua, T.\& G.)

Heracleum, $\mathbf{L}$.

lanatum, Michx.

Daucus, $\mathbf{L}$.

Carota, $L$.

\section{ARALIACE $刃$}

Aralia, L.

hispida, Vent.

nudicaulis, L.

var. prolifera, Apgar.

quinquefolia, (L.), Decne. \& Planch.

racemosa, $\mathrm{L}$.

spinosa, $L$.

trifolia, (L.), Decne. \& Planch.

\section{Cornus, L.}

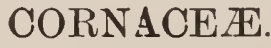

alternifolia, L. f.

Canadensis, L.

candidissima, Marsh. (C. paniculata, L'Hér.)

circinata, L'Hér. 
. 
florida, L.

sericea, $\mathrm{L}$.

stolonifera, Michx.

Nyssa, I.

sylvatica, Marsh. (N. multiflora, Wang.)

Sambucus, $\mathbf{I}$.

CAPRIFOLIACE

Canadensis, L.

var. laciniata, Gray.

racemosa, L. (S. pubens, Michx.)

Viburnum, L.

acerifolium, L.

dentatum, L.

lantanoides, Michx.

Lentago, L.

molle, Michx.

nudum, L.

var. cassinoides, (L.), T. \& G.

Opulus, L.

prunifolium, L.

pubescens, Pursh.

Triosteum, L.

angustifolium, $\mathrm{L}$.

perfoliatum, $\mathrm{L}$.

Symphoricarpos, Juss.

racemosus, Michx.

vulgaris, Michx.

\section{Linnæa, Gronov.}

borealis, $\mathrm{L}$.

Ionicera, L.

cærulea, L.

Caprifolium, L. (L. grata, Gray, Manual.)

ciliata, Mubl.

glauca, Hill. (L. parviftora, Lam.)

hirsuta, Eaton.

sempervirens, $\mathrm{L}$.

Tatarica, $I$.

Diervilla, Tourn.

tritida, Monch.

Cephalanthus, I.

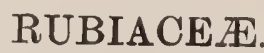

occidentalis, L. 
Oldenlandia, $\mathbf{I}$.

uniflora, L. (O. glomerata, Michx.)

Houstonia, I.

cærulea, L.

purpurea, L.

var. longifolia, (Gærtn.), Gray.

Mitchella, L.

repens, L.

Diodia, L.

teres, Walt.

Virginiana, L.

Galium, $\mathbf{L}$.

Aparine, L.

asprellum, Michx.

boreale, L.

circæzans, Michx.

con'cinnum, T. \& G.

lanceolatum, Torr.

latifolium, Michx.

Mollugo, $L$.

peregrinum, (Walt.) (G. hispidulum, Michx.)

pilosum, Ait.

var. puncticulosum, (Michx.), T. \& G.

trifidum, L.

var. latifolium, Torr.

var. pusillum, Gray.

triflorum, Michx.

verum, $I$.

\section{Valeriana, $\mathbf{L}$.}

VALERIANE无.

officinalis, $L$.

pauciflora, Michx.

sylvatica, Banks.

Valerianella, Tourn. (Fedia, Gcertn.)

olitoria, Poll.

radiata, Dufr.

Woodsiana, (T. \& G.), Walp.

var. patellaria, (Sulliv.), Gray.

var umbilicata, (Sulliv.), Gray.

DIPSACE $\mathrm{E}$.

Dipsacus, I.

fullonum, $L$.

sylvestris, Mill. 
$$
\text { . }
$$ 
COMPOSITE.

Vernonia, Schreb.

Noveboracensis, (L.), Willd.

Sclerolepis, Cass.

uniflora, (Walt.) (S. verticillata, Cass.)

Eupatorium, L.

ageratoides, L. f.

album, L.

var. subvenosum, Gray.

aromaticum, L.

var. melissoides, (Willd.), Gray.

cœlestinum, L. (Conoclinium colestinum, DC.)

foeniculoides, Walt. (E.f(eniculaceum, Willd.)

hyssopifolium, L.

leucolepis, T. \& G.

perfoliatum, L.

var. truncatum, Gray.

purpureum, L.

var. maculatum, (L.), Dar].

resinosum, Torr.

rotundifolium, L.

var. pubescens, (Muh].) (E. pubescens, Muhl.)

sessilifolium, L.

teucriifolium, Willd.

Mikania, Willd.

scandens, (L.), Willd.

\section{Kuhnia, I.}

eupatorioides, L.

Liatris, Schreb.

graminifolia, Pursh.

var. dubia, (Bart.), Gray.

scariosa, (L ), Willd.

spicata, (L.). Willd.

Chrysopsis, Nutt.

falcata, (Pursh), Ell.

Mariana, (L.), Nutt.

Bigelo via, DC.

nudata, (Michx.), DC.

Solidago, I.

var. virgata, (Nutt.), T. \& G.

arguta, Ait. (S. Muhlenbergii, T. \& G.)

bicolor, L. 
var. hispida, (Muh].) (var. concolor, T.\& G.)

crsia, L.

Canadensis, L.

var. procera, (Ait.), T. \& G.

var. scabra, (Muhl.), T. \& G.

Caroliniana, (L.) (S. temuifolia, I'ur'sh).

Elliottii, T. \& G. (S. elliptica, Gray, not Ait.)

juncea, Ait. (S. arguta, T. \& G., not Ait.)

lanceolata, $\mathrm{L}$.

latifolia, L.

macrophylla, Pursh. (S. thyrsoidea, E. Meyer.)

neglecta, T. \& G.

var. uniligulata, (Nutt.) (S. linoides, T. \& G., not Soland.)

nemoralis, Ait.

odora, Ait.

var. inodora, Gray.

patula, Muhl.

pilosa, Walt.

puberula, Nutt.

rigida, $\mathrm{L}$.

rugosa, Mill. (S. altissima, T. \& G., not $L$.)

sempervirens, L.

serotina, Ait. (S. gigantea, Gray, Manual.)

var. gigantea, (Ait.), Gray. (S. serotina, Gray, Manual.)

speciosa, Nutt.

var. angustata, T. \& G.

squarrosa, Muhl.

stricta, Ait. (S. virgata, Michx.)

uliginosa, Nutt. (S. stricta, T. d G., not Ait.)

ulmifolia, Muhl.

\section{Sericocarpus, Nees.}

asteroides, (L.) (S. conyzoides, Nees.)

linifolius, (L.) (S. solidagineus, Nees.)

\section{Aster, I.}

acuminatus, Michx.

amethystinus, Nutt.

concolor, L.

cordifolius, $\mathrm{L}$.

var. lævigatus, Porter.

cornifolius, Muhl. (Diplopappus cornifolius, Darl.)

corymbosus, Ait.

diffusus, Ait. (A.miser, T. d. G., not Ait., nor L.) 
dumosus, $\mathrm{L}$.

var. coridifolius, (Michx.), T. \& G.

ericoides, L.

gracilis, Nutt. (A. surculosus, Michx., var. gracilis, Gray.)

lievis, L.

linariifolius, L. (Diplopappus linariifolize, Hook.)

macrophyllus, L.

multiflorus, Ait.

nemoralis, Ait.

Noræ-Angliæ, L.

var. roseus, (Desf.), DC.

Novi-Belgii, L. (A. longifolius, Gray, Mrrnucal.)

var. elodes, (T. \& G.), Gray.

var. litoreus, Gray.

paniculatus, Lam. (A. simplex, Willd.)

patens, Ait.

var. phlogifolins, (Muhl.), Nees.

polyphyllus, Willd.

prenanthoides, Muhl.

puniceus, L.

radula, Ait.

sagittifolius, Willd.

salicifolius, Ait.

spectabilis, Ait.

subulatus, Michx. (A. linifolius, 'T. \& G., not L.)

surculosus, Michx.

tenuifolius, L. (A. flexuosus, Nutt.)

Tradescanti, L.

umbellatus, Mil]. (Diplopappus umbellatus, T.\& G.)

var. humilis, (Willd.) (Diplopappus anygdalinus, T. \& G.) undulatus, $\mathrm{L}$.

vimineus, Lam. (A. Tradescanti, T. \& G., not L.)

\section{Frigeron, $\mathbf{L}$.}

annuus, (L.), Pers.

bellidifolius, Mubl.

Canadensis, $\mathrm{L}$.

Philadelphicus, L.

ramosus, (Walt.) (E. strigosus, Muhl.)

var. discoideus, (Robbins).

Baccharis, $\mathrm{L}$.

halimifolia, L.

Pluchea, Cass.

camphorata, (L.), DC. (Includes P. foetida, DC.) 

fotida, (L.) (P. lififons, DC.)

Filago, L.

Crermanica, $T$.

Antennaria, Gartn.

plantaginifolia, (L.), Hoot.

Aliaphalis, $\mathbf{D C}$.

margaritacea. (L.), Benth. \& Hook. (Antennaria margaritacea, R. $B r$.

Gnap!lalinu, L.

decurrens, Ives.

obtusifolium, L. (G. polycephalum, Michx.)

purpureum, L.

uliginosum, L.

Inula, L.

Helenium, $T_{\text {. }}$

Polymuia, $\mathbf{T}$.

Canadensis, $\mathbf{L}$.

Uvedalia, L.

Silphium, L.

Iva, L.

perfoliatum, $I$.

frutescens, I.

Ambrosia, I.

artemisiafolia, $\mathrm{L}$.

trifida, I.

var. integrifolia, (Muhl.), T. \& G.

Xanthium, $\mathbf{L}$.

Canadense, Mill., var. echiuatum, (Murr.), Gray. (X. strumarium, I., var. echinatum, Gray.)

spinosum, $T$.

strumarium, $I$.

Helionsis, Pers.

helianthoides, (L.) (H. levis, Pers.)

Eclipta, I.

(ulba, (L.), Hassk. (E. procumbens, Michx.)

Rudbeckia, T.

fulgida, Ait.

hirta, $I$.

laciniata, $\mathrm{L}$.

speciosa, Wender.

triloba, $I$. 
Helianthus, $I_{1}$.

aigustifolius. $\mathrm{I}$.

cunnurcs, $I$.

decapetalus, L.

divaricatus, $\mathrm{L}$.

giganteus, L.

var. ambiguus, T. \& G.

strumosus, L.

tracheliifolius, Willd.

tuberosus, I. (Inclucles II. Cloronicoides, T. a (r., not Tr.mm.) Actinomeris, Nutt.

alternifolia, (L.), DC. (A. squariosa, Nutt.)

Coreopsis, $\mathbf{L}$.

bidentoides, Nutt.

discoidea, T. \& G.

lanceolata, $\mathrm{L}$.

rosea, Nutt.

trichosperma, Michx.

Birlens, I.

Beckii, Torr.

bipinnata, L.

cernua, L.

connata, Muhl.

frondosa, L.

licvis, (L.) (B. chrysanthemoides, Michx.)

Galinsoga, Ruiz e Par.

parviflora, Cav.

Helenimm, L.

autumnale, $\mathrm{L}$.

parviflorum, Nutt.

Achillea, $\mathbf{L}$.

Millefolium, $t$.

Anthemis, $\mathbf{L}$.

arvensis, $L$.

Cotula, L. (Maruta Cotula, DC.)

nobilis, $I$.

tinctoric, $L$.

Chrysanthemum, I.

Lercanthemum, I. (Lerucanthemum mulgare, Lam.)

I'arthenium, (L.), Per's.

Matricaria, I.

Chamomilla, $L$.

inoctora, $t$. 
Tanacetum, $\mathbf{T}$. vulgare, $L$.

Artemisia, L.

Absinthium, $L$.

biennis, Willd.

caudata, Michx.

vulgaris, $L$.

Tussilago, L.

Farfara, $L$.

Petasites, Gartn.

vulgaris, Desf.

Arnica, L.

acaulis, (Walt.) (A. nudicaulis, Nutt.)

Erechtites, Raf.

hieracifolia, (L.), Raf.

Senecio, $\mathbf{L}$.

aureus, $\mathrm{L}$.

var. Balsamitæ, (Muhl.), T. \& G.

var. obovatus, (Muhl.), T. \& G.

Cineraria, $D C$.

tomentosus, Michx.

vulgaris, $L$.

Cacalia, L.

atriplicifolia, $\mathrm{L}$.

reniformis, Muhl.

suareolens, L.

Arctium, $\mathrm{L}$.

Lappa, L. (Lappa officinalis, All.) var. tomentosum, (Pers.), Gray.

Carduus, L.

nutans, $L$.

Cnicus, L. (Cirsium, $D C$.)

altissimus, (L.), Willd.

var. discolor, (Muhl.), Gray. (C. discolor, spreng.)

arvensis, (L.), Hoffim.

lanceolatus, $\left(I_{\text {. }}\right)$, Hoffm.

muticus, (Michx.), Pursh.

odoratus, (Mubl.) (Cirsium pumilum, Spreng.)

spinosissimus, (Walt.), Darl. (C. horridulum, Michx.)

Onopordon, L.

acanthium, $L$.

Centaurea, $\mathrm{L}$.

Calcitrapa, L. 


$$
\text { . }
$$


Cyanus, $I$.

nigra, $I$.

\section{Cichorium, L.}

Intybus, $I$.

Krigia, Scllreb.

amplexicaulis, (Michx.), Nutt. (C'ynthia Viryinica, Don.)

Virgiuica, (L.), Willd.

Lapsana, L.

communis, $L$.

Crepis, I.

biennis, $I$.

virens, $I$.

tectorum, $I$.

Hieracium, $\mathbf{I}$.

aurantiacum, $L$.

Canadense, Michx.

Growovii, L.

Marianum, Willd.

murorum, $L$.

paniculatum, L.

scabrum, Michx.

venosum, $\mathrm{L}$.

Leontodon, L.

cutumnale, $L$.

'Muaxacum, Hall.

o.fficinale, Web. ( T. Dens-leonis, Desf.)

Lactuca, L. (Includes Mulgedium, Cass.)

Canadensis, L.

Floridana, (L.), Grertn.

hirsuta, Muhl. (I. Cunaclensis, I., var. sangrinea, T' \& G.)

integrifolia, Bigel. (I. Canadensis, I., var. integr fifolia, Gray.)

leucopbrea, (Willd.), Gray. (II. leucophcum, DC:)

Scceriola, $L$.

villosa, (Jacq.) (MI. acuminatum, $D C_{.}^{\gamma}$ )

Prenanthes, I. (Includes Nabalus, Cass.)

alba, L.

altissima, L.

autumnalis, Walt. ( $N$. virgatus, $\left.D C_{.}\right)$

racemosa, Michx.

var. pinnatificla, Gray.

serpentaria, Pursh. (N. Fraseri, $D\left(C_{*}\right)$

\section{Sonchus, L.}

arvensis, $L$. 

asper, Vilt.

oleraceus, $I$.

Tragopogon, $\mathbf{T}$.

porrifolius, $L$.

pratensis, $L$.

Lobelia, I.

CAMPANULACE $\nexists$ E.

Canbyi, Gray.

cardinalis, $\mathrm{L}$.

Dortmanna, L.

inflata, L.

Kalmii, L.

Nuttallii, Rœm. \& Schult.

puberula, Michx.

spicata, Lam.

syphilitica, L.

Campanula, L.

Americana, L.

aparinoides, Pursh.

glomerata, $L$.

rapunculoides, $\mathcal{L}$.

rotundifolia, $\mathrm{L}$.

Specularia, Heist.

perfoliata, (L.), A. DC.

Gaylussacia, HBK.

VACCINIACEA.

dumosa, (Andr.), T. \& G. var. hirtella, (Ait.), Gray.

frondosa, (L.), T. \& G.

resinosa, (Ait.), T. \& G.

Vaccinium, L.

Canadense, Kalm.

corymbosum, L.

var. amœnum, (Ait.), Gray.

var. disocarpum, (Bigel.) (var. atrococcum, Gray.)

Pennsylvanicum, Lam.

stamineum, L.

vacillans, Soland.

Oxycoccus, Pers.

macrocarpus, Pers. (V.macrocarpon, Ait.)

palustris, Pers. ( $V$. Oxycoccus, L.)

Chiogenes, Salisb.

bispidula, (L.), T. \& G. 



\section{ERICACE正.}

Arctostaphylos, Adans.

Uva-ursi, (L.), Spreng.

Gaultheria, L.

procumbens, L.

Cassandra, Don.

calyculata, (L.), Don.

Leucothoë, Don.

racemosa, (L.), Gray.

Epigaea, L.

repens, I.

Andromeda, L.

ligustrina, Muhl.

Mariana, L.

Polifolia, L.

Kalmia, $\mathbf{L}$.

angustifolia, L.

glauca, Ait.

latifolia, L.

Leiophyllum, Pers.

buxifolium, (Berg.), Ell.

Ledum, $\mathbf{L}$.

latifolium, Ait.

Rhododendron, $\mathbf{L}$.

Canadense, (L.) (Rhodora Canadensis, L.)

maximum, $\mathrm{L}$.

nudiflorum, (L.), Torr. (Azalea nudiflora, L.)

viscosum, (L.), Torr. (A. viscosa, $L$.)

var. glaucum, (Lam.), Gray.

var. nitidum, (Pursh), Gray.

Pyrola, L.

chlorantha, Sw.

elliptica, Nutt.

oxypetala, Austin.

rotundifolia, L.

var. asarifolia, (Michx.), Hook.

secunda, L.

Moneses, Salisb.

uniflora, (L.), Gray.

Chimaphila, Pursh.

maculata, (L.), Pursh.

umbellata, (L.), Nutt. 
Clethra, L.

alnifolia, $\mathrm{L}$.

Pterospora, Nutt.

MONOTROPE正.

andromedea, Nutt.

Monotropa, $\mathbf{L}$.

uniflora, I.

Hypopitys, I.

Monotropa, Crantz. (M. Hypopitys, L.)

DIAPENSIACE无.

Pyxidanthera, Michx.

barbulata, Michx.

PLUMBAGINE $\nexists$.

Statice, $\mathbf{L}$.

Limonium, L., var. Caroliniana, Gray.

PRIMULACE正.

Hottonia, I. inflata, Ell.

Lysimachia, $\mathbf{L}$.

nummularia, $L$.

quadrifolia, L.

terrestris, (L.) (L. stricta, Ait.)

thyrsiflora, $\mathbf{L}$.

vulgaris, $L$.

Steironema, Raf.

ciliatum, (L.), Raf. (Lysimachia ciliata, $L$.)

lanceolatum, (Walt.), Gray. (L. lanceolata, Walt.)

var. angustifolium, (Lam.), Gray.

var. hybridum, (Michx.), Gray.

Trientalis, L.

Americana, (Pers.), Pursh.

Anagallis, L.

arvensis, $L$.

Samolus, L.

Valerandi, $L$.

var. floribundus, (HBK.) (var. Americanus, Gray.)

Diospyros, L.

EBENACE正.

Virginiana, L. 


\section{.}


OLEACE正。

Fraxinus, $\mathbf{L}$.

Americana, L.

pubescens, Lam.

sambucifolia, Lam.

viridis, Michx. f.

Chionanthus, $\mathbf{L}$.

Virginica, L.

Ligustrum, $\mathbf{L}$.

vulgare, $L$.

\section{APOCYNACEE.}

Vinca, L.

minor, $L$.

Apocynum, $\mathbf{L}$.

androsæmifolium, $\mathbf{L}$.

cannabinum, L.

var. hypericifolium, (Ait.), Gray.

var. pubescens, (R. Br.), DC.

ASCLEPIADE无.

Acerates, Ell.

viridiflora, (Raf.), Ell.

var. lanceolata, (Ives), Gray.

Asclepias, L.

exaltata, (L.), Muhl. (A. phytolaccoides, Pursh.)

incarnata, $\mathrm{L}$.

var. pulchra, (Ehrh.), Pers.

lanceolata, Walt. (A. paupercula, Michx.)

obtusifolia, Michx.

purpurascens, $\mathrm{L}$.

quadrifolia, L.

rubra, L.

Syriaca, L. (A. Cornuti, Decne.)

tuberosa, $\mathrm{L}$.

variegata, $\mathrm{L}$.

verticillata, $\mathrm{L}$.

Vincetoxicum, Moench.

nigrum, (L.), Moench.

Gonolobus, Michx.

lævis, Michx., var. macrophyllus, (Michx.), Gray.

obliquus, (Jacq.), R. Br. 
Polypremum, L.

\section{LOGANIACE $\nexists$ E.}

procumbens, $L$.

Erythræa, Rich.

\section{GENTIANE}

ramosissima, Pers. (Includes var. pulchella, Griseb.)

Sabbatia, Adans.

angularis, (L.), Pursh.

dodecandra, (L.) (S. chloroides, Pursh.)

gracilis, (Michx.), Salisb.

lanceolata, (Walt.), T. \& G.

stellaris, Pursh.

Gentiana, I.

alba, Muhl.

Andrewsii, Griseb.

angustifolia, Michx.

crinita, Frol.

linearis, Froel. (G. Saponaria, L., var. linearis, Gray.)

quinquefolia, L. (G. quinqueflora, Lam.)

Saponaria, L.

villosa, L. (G. ochroleuca, Froel.)

Halenia, Borkh. deflexa, (Smith), Griseb.

Obolaria, $\mathbf{L}$.

Virginica, L.

Bartonia, Muhl.

Virginica, (L.) (B. tenella, Muhl.)

Menyanthes, $\mathbf{L}$.

trifoliata, $\mathbf{L}$.

Limnanthemum, Gmel.

lacunosum, (Vent.), Griseb.

Phlox, L.

POLEMONIACE E.

divaricata, $\mathrm{L}$.

maculata, $\mathrm{L}$.

paniculata, $L$.

pilosa, $\mathbf{L}$.

reptans, Michx.

subulata, L.

Polemonium, $\mathbf{L}$.

cæruleum, L.

reptans, L. 


\section{HYDROPHYLLACE $\nexists$.}

Hydrophyllum, $\mathbf{L}$.

Canadense, $\mathrm{L}$.

Virginicum, L.

Ellisia, I.

Nyctelea, L. (Includes E. ambigua, Nutt.)

Heliotropium, I.

BORRAGINE正.

Europceum, $L$.

Cynoglossum, $\mathbf{L}$.

officinale, $L$.

Virginicum, $\mathrm{L}$.

Echinospermum, Sw.

Lappula, (L.), Lehm.

Virginianum, (L.) (Cynoglossum Morrisoni, DC.)

Asperugo, L.

procumbens, $L$.

Symphytum, L.

officinale, $L$.

Lycopsis, $\mathbf{L}$.

arvensis, $L$.

Mertensia, Roth.

Virginica, (L.), DC.

Myosotis, $\mathbf{L}$.

arvensis, $\left(L_{.}\right)$, Willd.

laxa, Lehm. (M. palustris, Relh., var. laxa, Gray.) palustris, Relh.

Virginica, (L.) (M. verna, Nutt.)

Onosmodium, Michx.

Virginianum, (L.), DC.

Lithospermum, $\mathbf{L}$.

arvense, $L$.

canescens, (Michx.), Lehm.

latifolium, Michx.

officinale, $L$.

Echium, L.

vulgare, $I$.

CONVOLVULACE正.

Ipomoea, $\mathbf{L}$.

coccinea, $L$.

Nil, (L.), Pursh, not Roth. 

pandurata, (L.), Meyer.

purpurea, (L.), Lam.

Convolvulus, $\mathbf{L}$.

arvensis, $L$.

sepium, L. (Calystegia sepium, R. Br., in part.)

var. Americanus, Sims. (Calys. sepium, R. Br., in part.)

var. repens, (L.), Gray. (var. pubescens, Gray.)

spithamæus, L. (Calys. spithamcea, Pursh.)

Breweria, R. Br.

Pickeringii, (Torr.), Gray. (Bonamia Pickeringii, Gray.)

Cuscuta, I.

arvensis, Beyrich.

Cephalanthi, Engelm. (C. tenuiflora, Engelm.).

compacta, Juss.

Coryli, Engelm. (C. inflexa, Engelm.)

Epilinum, Weihe.

Gronovii, Willd.

SOLANACE正.

Solanum, L.

Carolinense, L.

Dulcamara, $L$.

nigrum, $L$.

rostratum, Dun.

\section{Physalis, L.}

angulata, L.

Philadelphica, Lam.

pubescens, L.

Virginiana, Mill. (P. viscosa, Gray, not L.)

\section{Nicandra, Adans.}

physaloides, (L.), Grertn.

Lycium, $\mathbf{L}$.

vulgare, (Ait.), Dun.

Datura, L.

Stramonium, $L$.

Tatula, $L$.

Hyoscyamus, $\mathbf{L}$.

niger, $L$.

Nicotiana, $\mathbf{L}$.

rustica, $L$.

Petunia, L.

axillaris, (Lam.) (P.nyctaginiflora, Juss.) 


\section{.}


SCROPHULARINE无.

Verbascum, L.

Blattaria, $L$.

Lychnitis, $L$.

Thapsus, $L$.

Linaria, Juss.

Canadensis, (L.), Dumont.

Elatine, Mill.

genistifolia, (L.), Mill.

vulgaris, Mill.

Scrophularia, L.

nodosa, L., var. Marylandica, (L.), Gray.

Chelone, I.

glabra, L.

Pentstemon, Mitch.

hirsutus, (L.), Willd. (P.pubescens, Soland.)

lævigatus, Soland., var. Digitalis, (Nutt.), Gray. (P. Digitalis, Nutt.)

Mimulus, $\mathbf{L}$.

alatus, Soland.

moschatus, Dougl.

ringens, $\mathbf{L}$.

Conobea, Aubl.

multifida, (Michx.), Benth.

Herpestis, Gærtn, f.

Caroliniana, (Walt.) (H. amplexicaulis, Pursh.) (Southern N. J. ?)

Gratiola, L.

aurea, Muhl.

pilosa, Michx.

sphærocarpa, Ell.

Virginiana, $\mathbf{L}$.

\section{Ilysanthes, Raf.}

gratioloides, (L.), Benth.

Micranthemum, Michx.

Nuttallii, Gray.

Limosella, L.

aquatica, L., var. tenuifolia, (Nutt.), Hoffm.

Veronica, $\mathbf{L}$.

agrestis, $L$.

Americana, Schwein.

Anagallis, L. 

var. latifolia, Britton.

arvensis, $I$.

Byzantina, (Sibth. \& Smith.) (V. Buxbaumii, Ten.)

Chamoedrys, $I$.

hedercefolia, $L$.

officinalis, $\mathbf{L}$.

peregrina, L.

scutellata, L.

serpyllifolia, L.

Virginica, L.

Buchnera, L.

Americana, L.

Gerardia, L.

auriculata, Michx.

flava, $\mathbf{L}$.

maritima, Raf.

pedicularia, $\mathrm{L}$.

purpurea, L.

var. paupercula, Gray.

tenuifolia, Vahl.

Virginica, (L.) (G. quercifolia, Pursh.)

Castilleja, L. f.

coccinea, (L.), Spreng.

Schwalbea, $\mathbf{L}$.

Americana, L.

Pedicularis, $\mathbf{L}$.

Canadensis, L.

lanceolata, Michx.

Rhinanthus, L.

Crista-galli, L.

Melampyrum, $\mathbf{L}$.

lineare, Lam. (M. Americanum, Michx.)

Aphyllon, Mitch.

OROBANCHACE正.

uniflorum, (L.), Gray.

Orobanche, L.

minor, $L$.

Conopholis, Wallr.

Americana, (L. f.), Wallr.

Epiphegus, Nutt.

Virginiana, (L.), Bart.

var. Rauana, Austin. 


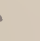


Utricularia, $\mathbf{I}$.

\section{LENTIBULARIEÆ.}

clandestina, Nutt.

cornuta, Michx.

fibrosa, Walt. (U. striata, Le Conte.)

gibba, L.

inflata, Walt.

intermedia, Hayne.

minor, $\mathrm{L}$.

purpurea, Walt.

- resupinata, B. D. Greene.

subulata, L.

var. cleistogama, Gray.

vulgaris, L. (Includes var. Americana, Gray.)

Catalpa, Juss.

BIGNONIACEA.

bignonioides, Walt. (Native within the circle?)

Tecoma, Juss.

radicans, (L.), Juss.

Martynia, I.

PEDALINE正.

proboscidea, Glox.

ACANTHACE王.

Ruellia, I. ciliosa, Pursh, var. hybrida, (Pursh), Gray.

Dianthera, I.

Americana, L.

Phryma, I.

VERBENACEA.

Leptostachya, L.

Lippia, $\mathbf{L}$.

lanceolata, Michx.

Verbena, $\mathbf{L}$.

angustifolia, Michx.

bracteosa, Michx.

hastata, L.

officinalis, $L$.

stricta, Vent.

urticæfolia, I.

Collinsonia, $\mathbf{I}$.

LABIAT $\mathrm{E}$.

Canadensis, L. 

Mentha, $\mathbf{L}$.

aquatica, L. (Inclucles var. glabrata, Benth.) var. crispa, (L.), Benth.

arvensis, $L$.

Canadensis, $\mathrm{L}$.

var. borealis, (Michx.), Wood. (var. glabrata, Benth.) piperita, $L$.

var. subhirsuta, Benth.

rotundifolia, $L$.

sativa, $L$.

sylvestris, $L$.

var. alopecuroides, (Hull), Baker.

viridis, $L$.

\section{Lycopus, L.}

Europceus, $L$.

rubellus, Mœnch. (L. Europceus, L., var. integrifolius, Gray.) sessilifolius, Gray. (L. Europceus, I., var. sessilifolius, Gray.) sinuatus, Ell. (L. Europceus, L., var. sinuatus, Gray.) Virginicus, L.

\section{Cunila, $\mathbf{L}$.}

Mariana, I.

\section{Pycnanthemum, Michx.}

aristatum, Michx.

clinopodioides, T. \& G.

flexuosum, (Walt.) (P. linifolium, Pursh.)

incanum, (L.), Michx.

muticum, (Michx.), Pers.

Torreyi, Benth.

Virginicum, (L.) (P. lanceolatum, Pursh.)

\section{Origanum, $\mathbf{L}$.}

vulgare, $L$.

\section{Thymus, I.}

Serpyllum, $L$.

\section{Satureia, L.}

hortensis, $L$.

Hyssopus, $\mathbf{L}$.

officinalis, $L$.

Hedeoma, Pers.

pulegioides, (L.), Pers.

Calamintha, Monch.

Clinopodium, Benth.

Melissa, $\mathbf{I}$.

officinalis, $L$. 
Salvia, L.

lyrata, L.

Monarda, I.

didyma, L.

fistulosa, $\mathrm{L}$.

punctata, L.

Blephilia, Raf.

ciliata, (L.), Raf.

hirsuta, (Pursh), Benth.

Lophanthus, Benth.

nepetoides, (L.), Benth.

scrophulariæfolius, (Willd.), Benth.

Nepeta, $\mathbf{L}$.

Cataria, $L$.

hederacea, (L.) (N. Glechoma, Benth.)

Scutellaria, $\mathbf{L}$.

cordifolia, Muhl. (S. versicolor, Nutt.)

galericulata, L.

integrifolia, $\mathrm{L}$.

lateriflora, L.

nervosa, Pursh.

parvula, Michx.

pilosa, Michx.

var. ovalifolia, (Pers.), Benth.

serrata, Andr.

Brunella, I.

vulgaris, $L$.

Physostegia, Benth.

Virginiana, (L.), Benth.

Marrubium, $\mathbf{L}$.

vulgare, $L$.

Stachys, I.

arvensis, $L$.

aspera, Michx. (S. palustris, L., var. aspera, Gray.)

cordata, Ridd. (S. palustris, L., var. cordata, Gray.)

hyssopifolia, Michx.

palustris, $\mathrm{L}$.

Galeopsis, I.

Ladanum, $L$.

Tetrahit, $I$.

Leomurus, I.

Cardiaca, $L$. 
Marrubiastrum, $L$.

Sibiricus, $t$.

Lamium, $\mathbf{L}$.

album, $L$.

amplexicaule, $L$.

purpureum, $\boldsymbol{L}$.

Ballota, I.

nigra, $L$.

Trichostema, L.

dichotomum, L.

lineare, Nutt.

Isanthus, Michx.

brachiatus, (L.) (I. cceruleus, Michx.)

Teucrium, L.

Canadense, L.

occidentale, Gray.

Ajuga, I.

reptans, $L$.

\section{Plantago, $\mathbf{L}$.}

PLANTAGINE无.

cordata, Lam.

decipiens, Barn. (P. maritima, L., var. juncoides, Gray.)

elongata, Pursh. (P. pusilla, Nutt.)

heterophylla, Nutt.

lanceolata, $L$.

major, $L$.

var. minima, (DC.), Decne

Patagonica, Jacq., var. aristata, (Michx.), Gray.

Rugelii, Decne. (P. Kamtschatica, Gray, not Cham.)

Virginica, L.

Anychia, Rich.

ILLECEBRACE无.

Canadensis, (L.) (A. capillacea, $D C$.)

dichotoma, Michx.

Scleranthus, $\mathbf{C}$.

annurus, $L$.

Amarantus, $\mathbf{I}$.

AMARANTACEAE.

albus, $L$.

Blitum, $L$.

chlorostachys, Willd. (A. retroflexus, L., var. chlorostachys, Gray.)

hybridus, L. (A. retroflexus, L., var. hybridus, Gray.) 
hypochondriacus, $I$.

paniculatus, $L$.

pumilus, Raf.

retroflexus, $L$.

spinosus, $L$.

viridis, $L$.

Acnida, L.

cannabina, L.

tuberculata, Moq. (Montelia tamariscina, Gray, in part.)

Cycloloma, Moq.

\section{CHENOPODIACE}

platyphyllum, (Michx.), Moq.

Chenopodium, I.

album, $L$.

ambrosioides, $L$.

var. anthelminticum, (L.), Gray.

Bonus-Henricus, I. (Blitum Bonus-Henricus, Reichenb.)

Boscianum, Moq. (C. album, L., var. Boscianum, Gray.)

Botrys, $L$.

capitatum, (L.), S. Wats. (B. capitatum, L.)

glancum, $I$.

hybriclum, $L$.

leptophyllum, Nutt.

murale, $I$.

rubrum, L. (B. maritimum, Nutt.)

urbicum, $L$.

var. rhombifolium, (Muhl.), Moq.

Roubieva, Moq.

multifida, (L.), Moq. (C. multifidum, L.)

\section{Atriplex, I.}

arenaria, Nutt.

patula, L., var. hastata, (L.), Gray.

var. littoralis, (L.), Gray.

rosea, $L$.

\section{Salicornia, L.}

ambigua, Michx. (S. fruticosa, L., var. ambigua, Gray.) herbacea, $\mathrm{L}$.

mucronata, Bigel. (S. Virginica, Gray, not L.)

Suæda, Forsk.

linearis, (Ell.), Torr., var. salsa, (Michx.) (S. maritima, Gray, not L.) 
Salsola, L.

Kali, L.

\section{PHYTOLACCACE无.}

Phytolacca, L.

decandra, L.

\section{POLYGONACEZ.}

\section{Polygonum, L.}

acre, HBK.

amphibium, L. (Includes var. aquaticum, Willd.)

arifolium, $\mathrm{L}$.

articulatum, $\mathrm{L}$.

aviculare, $\mathrm{L}$.

Careyi, Olney.

cilinode, Michx.

Convolvulus, $I$.

cuspidatum, Sieb. \& Zucc.

dumetorum, L., var. scandens, (L.), Gray.

erectum, L. (P. aviculare, I., var. erectum, Roth.)

Hartwrightii, Gray.

Hydropiper, $I$.

hydropiperoides, Michx.

incarnatum, Ell.

maritimum, L.

orientale, $I$.

Pennsylvanicum, L.

Persicaria, $L$.

ramosissimum, Michx.

sagittatum, I.

tenue, Michx.

terrestre, (Willd.) (P. amphibium, L., var. terrestre, Willd.)

Virginianum, L.

\section{Fagopyrum, Grertn.}

esculentum, Moench.

\section{Rumex, L.}

Acetosella, $L$.

altissimus, Wood. (R. Britannica, Gray, not I.)

Britannicus, L. (P. orbiculatus, Gray.)

conglomeratus, Murr.

crispus, $L$.

hastatulus, Baldw. (R. Engelmanni, Ledeb.)

maritimus, L.

obtusifolius, $\mathcal{L}$. 

Patientia, I.

verticillatus, $L$.

PODOSTEMACE无.

Podostemon, Michx.

ceratophyllum, Michx.

ARISTOLOCHIACE $巴$.

Asarum, $\mathbf{L}$.

Canadense, L.

Aristolochia, $\mathbf{I}$.

Serpentaria, L.

PIPERACEA.

Saururus, L.

cernuus, L.

LAURINEA.

Sassafiras, Nees.

officinale, Nees.

Lindera, Thunb.

Benzoin, (L.), Meisn.

\section{THYMEL 玵ACE无.}

Dirca, L.

palustris, L.

LORANTHACEAE.

\section{Arceuthobium, Rich.}

pusillum, Peck.

Phoradendron, Nutt.

flavescens, (Pursh), Nutt.

SANTALACE无.

Comandra, Nutt.

umbellata, (L.), Nutt.

EUPHORBIACE王.

Euphorbia, I.

corollata, L.

Cyparissias, $I$.

Darlingtonii, Gray.

Helioscopia, $L$.

hypericifolia, L.

Ipecacuanhæ, L.

Iathyris, $I$. 

maculata, $\mathrm{L}$.

polygonifolia, L.

Phyllanthus, $\mathbf{I}$.

Carolinensis, Walt.

Croton, L.

capitatus, Michx.

Crotonopsis, Michx.

linearis, Michx.

Acalypha, $\mathbf{L}$.

Caroliniana, Walt.

Virginica, L.

var. gracilens, (Gray), Muell. Arg.

URTICACE 现.

\section{Ulmus, L.}

Americana, L.

fulva, Michx.

racemosa, Thomas.

Celtis, L.

occidentalis, L.

var. pumila, (Pursh), Gray.

Humulus, I.

Lropulus, $L$.

Cannabis, $\mathbf{L}$.

sativa, $L$.

Broussonetia, Vent.

papyrifera, (L.), Vent.

Morus, $\mathbf{L}$.

alba, $L$.

rubra, L.

Urtica, I.

dioica, $L$.

gracilis, Ait.

urens, $L$.

Laportea, Gandich.

Canadensis, (L.), Gaudich.

Pilea, Lindl.

pumila, (L.), Gray.

Boehmeria, Jacr.

cylindrica, (L.), Willd.

var. scabra, Porter.

Parietaria, L.

Pennsylvanica, Muhl. 


\section{PLATANACE $\nexists$.}

Platanus, L.

occidentalis, L.

Carya, Nutt.

JUGLANDEA.

alba, (L.) (C. tomentosa, Nutt.)

glabra, (Mill.), Torr. (C.porcina, Nutt.)

microcarpa, Nutt.

minima, (Marsh.) (C. amara, Nutt.)

ovata, (Mill.) (C. alba, Nutt.)

sulcata, (Willd.), Nutt.

Juglans, L.

cinerea, $\mathrm{L}$.

nigra, $\mathrm{L}$.

\section{MYRICACE正。}

Myrica, I.

asplenifolia, (L.), Banks. (Comptonia asplenifolia, Ait.) cerifera, $\mathrm{L}$.

Gale, L.

CUPULIFER丑.

Betula, L.

alba, L., var. populifolia, (Marsh.), Spach.

lenta, L.

lutea, Michx. f.

nigra, $\mathrm{L}$.

papyrifera, Marsh. (B. papyracea, Ait.)

pumila, L.

Alnus, Gærtn.

incana, (L.), Willd.

glutinosa, Willd.

serrulata, Willd.

Carpinus, L.

Caroliniana, Walt. (C. Americana, Michx.)

Ostrya, Scop.

Virginiana, (Mill.) (O. Virginica, Willd.)

Corylus, L.

Americana, Walt.

rostrata, Ait.

Quercus, L.

alba, L.

bicolor, Willd.

coccinea, Wang. 
falcata, Michx.

heterophylla, Michx. f.

ilicifolia, Wang.

imbricaria, Michx.

macrocarpa, Michx.

Muhlenbergii, Engelm. (Q. Prinus, L., var. acuminata, Michx.) var. humilis, (Marsh.), Britton. (Q. prinoides, Willd.)

nigra, L.

palustris, Du Roi.

Phellos, L.

Prinus, L. (Includes var. monticola, Michx.)

rubra, L.

Rudkinii, Britton.

stellata, Wang. (Q. obtusiloba, Michx.)

tinctoria, Bartr. (Q. coccinea, Wang., var. tinctoria, Gray.)

Castanea, Gartn.

pumila, Mill.

vulgaris, Lam., var. Americana, (Michx.), DC. (C.vesca, Grertn., var. Americana, Michx.)

\section{Fagus, L.}

ferruginea, Ait.

SALICINEA.

Salix, $\mathbf{L}$.

alba, $L$.

var. vitellina, $\left(L_{\text {. }}\right)$, Koch.

Babylonica, $L$.

candida, Willd.

cordata, Muhl.

var. angustata, (Pursh), Anders.

discolor, Muhl.

fragilis, $L$.

humilis, Muhl.

longifolia, Muhl.

lucida, Muhl.

myrtilloides, $\mathrm{L}$.

nigra, Marsh.

petiolaris, Smith.

purpurea, $L$.

rostrata, Richards. (S.livida, Wahl., var. occidentalis, Gray.) sericea, Marsh.

tristis, Ait.

viminalis, $L$. 
Populus, I.

alba, $L$.

balsamifera, L.

var. candicans, (Ait.), Gray.

dilatata, $L$.

grandidentata, Michx.

heterophylla, L.

monilifera, Ait. (Includes P. angulata, Ait.)

nigra, $L$.

tremuloides, Michx.

EMPETRACE E.

Corema, Don.

Conradii, Torr.

CERATOPHYLLEE.

Ceratophyllum, I.

demersum, $\mathbf{L}$.

var. echinatum, Gray.

HYDROCHARIDEA.

\section{Elodea, Michx.}

Canadensis, Michx. (Anacharis Canadensis, Planch.)

Vallisneria, L.

spiralis, L.

Limnobium, Rich.

Spongia, Ricb.

Microstylis, Nutt.

ORCHIDEÆ.

monophylla, (L.), Lindl.

unifolia, (Michx.) (M. ophioglossoicles, Nutt.)

Liparis, Rich.

liliifolia, (L.), Rich.

Lœselii, (L.), Rich.

Aplectrum, Nutt.

spicatum, (Walt.) (A. hiemale, Nutt.)

Corallorhiza, R. Br.

innata, $\mathrm{R}$. Br.

multiflora, Nutt.

odontorhiza, (Sw.), Nutt.

Tipularia, Nutt. unifolia, (Muhl.) ('T'. discolor, Nutt.)

Tistera, R. Br. australis, Lindl. 

convallarioides, (Sw.), Nutt.

cordata, (L.), R. Br.

\section{Spiranthes, Rich.}

cernua, (L.), Rich.

gracilis, Bigel.

graminea, Lindl., var. precox, (Walt.) (var. Walteri, Gray.)

latifolia, Torr.

simplex, Gray.

Goodyera, R. Br.

pubescens, (Willd.), R. Br.

repens, (L.), R. Br.

Arethisa, $\mathbf{L}$.

bulbosa, L.

Calopogon, R. Br.

tuberosus, (L.) (C.pr.lchellus, R. Br.)

\section{Pogonia, Juss.}

affinis, Austin.

divaricata, (L.), R. Br.

ophioglossoides, (L.), Ker.

trianthophorus, (Sw.) (P.pendula, Lindl.)

verticillata, (Willd.), Nutt.

\section{Orchis, L.}

rotundifolia, Pursh. (Habenaria rotundifolia, Richards.)

spectabilis, L.

\section{Habenaria, Willd.}

blephariglottis, (Willd.), Torr.

ciliaris, (L.), R. Br.

cristata, (Michx.), R. Br.

dilatata, (Pursh), Gray.

fimbriata, (Ait.), R. Br.

flava, (L.), Gray. (H. virescens, Spreng.)

Hookeriana, Torr. (H. Hookeri, 'T'orr.)

var. oblongifolia, Paine.

hyperborea, (L.), R. Br.

integra, (Nutt.), Spreng.

lacera, (Michx.), R. Br.

orbiculata, (Pursh), Torr.

peramœna, Gray.

psycodes, (L.), Gray.

tridentata, (Willd.). Hook.

viridis, (Sw.), R. Br., var. bracteata, (R. Br.), Reichenb.

Cypripedium, $\mathbf{L}$.

acaule, Ait. 


$$
\text { . }
$$


arietinum, $\mathrm{R}$. Br.

candidum, Muhl.

parviflorum, Salisb.

pubescens, Willd.

spectabile, $\mathrm{S} w$.

HAMODORACE正.

Lachnanthes, Ell.

tinctoria, (Walt.), El].

Cophiola, Ker.

tomentosa, (Muhl.) (L. aurea, Ker.)

Aletris, L.

aurea, Walt.

farinosa, $\mathrm{L}$.

IRIDEÆ.

Iris, $\mathbf{L}$.

versicolor, L.

prismatica, Pursh. (I. Virginica, Gray, not L.)

Belamcanda, Adans.

Chinensis, (L.), Red. (Pardanthus Chinensis, Ker.)

Sisyrinchium, $\mathbf{L}$.

angustifolium, Mill. (S. Bermudiana, Gray, not L.)

mucronatum, Michx. (S. Bermudiana, var. mucronatum, Gray.)

AMARYLLIDE坐.

Hypoxys, I.

erecta, L.

DIOSCOREACE无.

Dioscorea, I.

villosa, L.

LILIACE正.

Smilax, L.

bona.nox, L. (S.tamnoides, L.)

glauca, Walt.

herbacea, L.

var. pulverulenta, (Michx.), Gray.

hispida, Muhl.

laurifolia, L.

Pseudo-China, L.

rotundifolia, L.

tamnifolia, Michx.

Walteri, Pursh.

Asparagus, L.

officinalis, $L$. 
Polygonatum, Adans.

biflorum, (Walt.), Ell.

commutatum, (Schult.), Dietr. (P. giganteum, Dietr.)

Streptopus, Michx.

amplexifolius, (L.), DC.

roseus, (Pers.), Nichx.

Smilacina, Desf.

racemosa, (L.), Desf.

stellata, (L.), Desf.

trifolia, (L.), Desf.

Maianthemum, Wiggers.

Canadense, Desf. (S. bifolia, Ker, var. Canadensis, Gray.)

Convallaria, $\mathbf{L}$.

majalis, $L$.

Hemerocallis, L.

fulva, $L$.

Allium, I.

Canadense, Kalm.

tricoccum, Ait.

vineale, $L$.

Muscari, Mill.

botryoicles, (L.), Mill.

racemosum, (L.), Mill.

Ornithogalum, L.

nutans, $L$.

umbellatum, $L$.

Lilium, L.

Canadense, $\mathrm{L}$.

Philadelphicum, L.

superbum, $\mathrm{L}$.

Erythronium, $\mathbf{L}$.

albidum, Nutt.

Americanum, Ker.

Narthecium, Moehr.

Americanum, Ker. (N. ossifragum, Huds., var. Americanum, Gray.)

Chamalirium, Willd.

luteum, (L.), Gray.

Xerophyllum, Michx.

asphodeloides, (L.), Spreng.

Helonias, L.

bullata, L. 


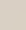


Tofieldia, Huds. racemosa, (Walt.) ('T. pubens, Pers.)

Uvularia, L.

grandiflora, Smith. (Includes U. flava, Smith.) perfoliata, L.

Oakesia, S. Wats. sessilifolia, (L.), S. Wats.

Clintonia, Raf. borealis, (Ait.), Raf.

Medeola, $\mathrm{L}$.

Virginica, L.

Trillium, I.

cernuum, L.

erectum, L.

erythrocarpum, Michx.

grandiflorum, (Michx.), Salisb.

Melanthium, $\mathbf{L}$.

latifolium, Desrouss.

Virginicum, L.

Veratrum, $\mathbf{L}$.

viride, Ait.

Zygadenus, Michx.

leimanthoides, Gray.

Amianthium, Gray.

muscretoxicum, (Walt.), Gray.

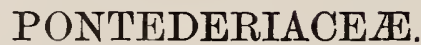

Pontederia, L.

cordata, L.

var. angustifolia, Pursh.

Heteranthera, Ruiz \& Pav.

graminea, (Michx.), Vahl. (Schollera graminea, Willd.) reniformis, Ruiz \& Pav.

\section{XYRIDE无.}

Xyris, $\mathrm{L}$.

Caroliniana, Walt.

fimbriata, Ell.

flexuosa, Muhl.

var. pusilla, Gray.

torta, Smith. 


\section{,}


Commelina, L.

\section{COMMELINACE王.}

communis, $L$.

hirtella, Vahl.

nudifiora, $T$.

Virginica, L.

Tradescantia, I.

Tirginica, $L$.

\section{Juncus, I.}

\section{JUNCACE $\nexists$.}

acuminatus, Michx. (.T. acuminatus, Michx., var. legitimus, Engelm.)

var. debilis, (Gray), Engelm.

articulatus, $\mathrm{L}$.

var. obtusatus, Engelm.

asper, Engelm.

Balticus, Dethard, var. littoralis, Engelm. (Westchester Co.?) bufonius, $\mathrm{L}$.

Canadensis, J. Gay. (J. Canadensis, J. Gay, var. longicaudatus, Engelm.)

var. brachycephalus, Engelm.

var. coarctatus, Engelm.

var. subcaudatus, Engelm.

dichotomus, Ell.

effusus, L.

var. conglomeratus, (L.), Engelm.

Gerardi, Lois.

Greenii, Oakes \& Tuck.

marginatus, Rostk.

var. biflorus, (Ell.), Engelm.

var. paucicapitatus, Engelm.

maritimus, Lam.

militaris, Bigel.

nodosus, I.

var. megacephalus, Torr.

pelocarpus, E. Meyer.

var. fluitans, (Michx.) (var. (?) subtilis, Engelm.)

Romerianus, Scheele. (Nevo Jersey?)

scirpoides, Lam. (.T. scirpoides, Lam., var. macrostemon, En gelm.)

tenuis, Willd.

var. secundus, (Poir.), Engelm.

trifidus, I. 


\section{.}


Luzula, DC.

campestris, (L.), DC.

pilosa, (L.), Willd.

spadicea, DC., rar. melanocarpa, (Desv.), E. Meyer. (L. parviflora, Desv., var. melanocarpa, Gray.)

TYPHACE涩.

'Typha, L.

angustifolia, $\mathbf{L}$.

latifolia, L.

Sparganium, L.

androcladum, (Engelm.), Morong. (S. simplex, Huds., var. androcladum, Engelm.)

var. fluctuans, Morong. (S. simplex, Hiuds., var. fluitans, Engelm.)

eurycarpum, Engelm.

simplex, Huds. (Includes var. Nuttallii, Engelm.)

var. angustifolium, (Michx.), Engelm.

AROIDE正.

Arisæma, Mart.

Dracontium, (L.), Schott.

triphyllum, (L.), Torr.

Peltandra, Raf.

undulata, Raf.

Virginica, (L.), Raf.

Calla, L.

palustris, $\mathrm{L}$.

Orontium, L.

aquaticum, L.

Symplocarpus, Salisb.

fœtidus, (L.), Salisb.

Acorus, L.

Calamus, L.

LEMNACE正.

Lemna, $\mathbf{L}$.

minor, L.

var. obscura, Austin.

var. orbiculata, Austin.

perpusilla, Torr.

var. trinervis, Austin.

trisulca, L.

Valdiviana, Philippi. (L. Torreyi, Austin.) 

Spirodela, Schleid.

polyrhiza, (L.), Schleid. (Lemna polyrhiza, L.)

Wolffia, Horkel.

Columbiana, Karst.

ALISMACE $Æ$.

Alisma, L.

Plantago, L., var. triviale, (Pursh). (var. Americanum, Gray.)

Sagittaria, L.

calycina, Engelm.

var. spongiosa, Engelm.

graminea, Michx.

heterophylla, Pursh.

subulata, (Pursh). (S. pusilla, Nutt.)

variabilis, Engelm., var. angustifolia, Engelm.

var. diversifolia, Engelm.

var. gracilis, (Pursh), Engelm.

var. hastata, (Pursh), Engelm.

var. latifolia, (Willd.), Engelm.

var. obtusa, (Willd.), Engelm.

var. pubescens, (Muhl.), Engelm.

NAIADACE无.

Triglochin, L.

maritimum, $\mathrm{L}$.

palustre, $\mathrm{L}$.

Scheuchzeria, $\mathbf{L}$.

palustris, $\mathbf{L}$.

Potamogeton, L.

amplifolius, Tuck.

compressus, L.

crispus, $\mathbf{L}$.

fluitans, Roth. (P. lonchites, Tuck.)

gramineus, L. (Includes var. graminifolius, Fries, and var. heterophyllus, Fries.)

vaj. maximus, Morong.

var.(?) myriophyllus, Robbins.

hybridus, Michx.

lucens, $\mathrm{L}$.

natans, L.

Oakesianus, Robbins.

obtusifolius, Mart. \& Koch.

pauciflorus, Pursh. 


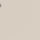


pectinatus, $\mathbf{L}$.

Pennsylvanicus, Cham. (P. Claytonii, Tuck.)

perfoliatus, L.

var. lanceolatus, Robbins.

prælongus, Wulf.

pulcher, Tuck.

pusillus, L. (Includes var. tenuissimus, Mart. \& Koch.)

Robbinsii, Oakes.

rufescens, Schrad.

Spirillus, Tuck.

Tuckermani, Robbins.

Vaseyi, Robbins.

Zizii, Mart. \& Koch. (P. lucens, L., var. minor, Nolte.)

zosteræfolius, Schum. (P. compressus, Fries, not I.)

Ruppia, L.

maritima, $\mathrm{L}$.

Zannichellia, L.

palustris, $\mathrm{L}$.

var. pedunculata, Gray.

Zostera, $\mathbf{L}$.

marina, $\mathrm{L}$.

Naias, $\mathbf{L}$.

flexilis, (Willd.), Rostk. \& Schmidt.

Indica, Cham., var. gracillima, R. Br.

ERIOCAULE无.

Eriocaulon, L.

decangulare, $\mathrm{L}$.

gnaphalodes, Michx.

septangulare, With.

\section{Cyperus, $\mathbf{L}$.}

\section{CYPERACE正.}

aristatus, Rottb. (C. inflexus, Muhl.)

compressus, $L$.

dentatus, Torr.

diandrus, Torr.

var. castaneus, (Bigel.), Torr.

Engelmanni, Steud.

erythrorhizos, Muhl.

esculentus, L. (C.phymatodes, Muhl.)

filiculmis, Vahl.

flavescens, $\mathrm{L}$. 
flavicomus, Vahl.

Grayii, Torr.

Lancastriensis, Porter.

Nuttallii, Eddy.

ovularis, (Vahl), Torr.

refractus, Engelm.

retrofractus, Engelm.

speciosus, Vahl. (C. Michauxianus, Gray, Manual.)

strigosus, L.

var. capitatus, Bockl.

var. compositus, Britton.

var. robustior, Kunth.

Torreyi, Britton.

Dulichium, Pers.

spathaceum, (L.), Pers.

Heleocharis, R. Br. (Eleocharis, R. Br.)

acicularis, (L.), R. Br.

diandra, C. Wright.

Engelmanni, Steud.

intermedia, (Muhl.), Schult.

melanocarpa, Torr.

olivacea, Torr.

ovata, (Roth), R. Br. (Eleocharis obtusa, Schult.)

palustris, (L.), R. Br.

var. calva, (Torr.), Gray.

var. glaucescens, (Willd.), Gray.

plantaginea, (Retz.), R. Br. (E. equisetoides, Torr.)

quadrangulata, (Michx.), R. Br.

Robbinsii, Oakes.

rostellata, Torr.

tenuis, (Willd.), Schult.

Torreyana, Bœckl. (E. microcarpa, Torr., var. filiculmis, Torr.)

tricostata, Torr.

tuberculosa, (Michx.), R. Br.

Dichromena, Michx.

cephalotes, (Walt.), Britton. (D. leucocephala, Michx.)

Psilocarya, Torr.

nitens, (Vahl), Britton. (Rhynchospora nitens, Gray.)

Fimbristylis, Vahl.

autumnalis, (L.), Rœm. \& Schult.

capillaris, (L.), Gray. 
castanea, Vahl. (F. spadicea, Vahl, var. castanea, Gray.) laxa, Vahl.

Vahlii, (Lam.), Link. (F. congesta, Torr.)

Scirpus, L.

atrovirens, Muhl.

debilis, Pursh.

fluviatilis, (Torr.), Gray.

lacustris, L. (S. validus, Vahl.)

lineatus, Michx.

maritimus, $\mathrm{L}$.

var. macrostachyos, Michx.

mucronatus, L.

Olneyi, Gray.

parvulus, Rœm. \& Schult. (Eleocharis pygmoea, T'orr.)

planifolius, Muhl.

polyphyllus, Vahl.

pungens, Vahl.

Smithii, Gray.

subterminalis, Torr.

sylvaticus, $\mathrm{L}$.

Torreyi, Olney.

Eriophorum, L.

alpinum, $\mathrm{L}$.

cyperinum, L. (Scirpus Eriophorum, Michx.)

var. laxum, (Gray). (S. Eriophorum, Michx., var. laxus, Gray.) gracile, Koch. (Includes var. paucinervium, Engelm.) polystachyon, L. (E. polystachyon, L., var. angustifolium, Gray.)

var. latifolium, (Hoppe), Gray.

vaginatum, $\mathrm{L}$.

Virginicum, L.

Fuirena, Rottb.

squarrosa, Michx.

var. pumila, Torr.

Hemicarpha, Nees.

micrantha, (Vahl), Britton. (H. subsquarrosa, Nees.)

Lipocarpha, R. Br.

maculata, (Michx.), Torr.

Rhynchospora, Vahl.

alba, (L.), Vahl.

axillaris, (Lam.), Britton. (R. cephalantha, Gray.)

capillacea, Torr. 


\section{-}


Curtisii, Steud. (R. pallida, M. A. Curtis.)

cymosa, (Willd.), Nutt.

fusca, (L.), Rœin. \& Schult.

glomerata, (L.), Vahl.

gracilenta, Gray.

Knieskernii, Carey.

macrostachya, Torr.

Torreyana, Gray.

Cladium, P. Br.

mariscoides, (Muhl.), Torr.

Scleria, Berg.

pauciflora, Mubl.

reticularis, Michx.

var. pubescens, Britton.

Torreyana, Walp. (S. laxa, Torr.)

triglomerata, Michx.

var. gracilis, Britton.

verticillata, Muhl.

\section{Carex, L.}

adusta, Boott.

æstivalis, M. A. Curtis.

alopecoidea, Tuck.

aperta, Boott.

aquatilis, Wahl.

arctata, Boott.

aurea, Nutt.

Barrattii, Schw. \& Torr.

bromoides, Schk.

bullata, Schk.

Buxbaumii, Wahl.

canescens, L.

var. brunnescens, (Pers.), Boott. (var. vitilis, Gray.)

Careyana, Torr.

cephalophora, Muhl.

conjuncta, Boott.

conoidea, Schk.

crinita, Lam.

var. gynandra, (Schw.), Schw. \& Torr.

Davisii, Schw. \& Torr.

debilis, Michx.

Deweyana, Schw.

digitalis, Willd. 
$$
\text { . }
$$ 
eburnea, Boott.

echinata, Murray. (C. stellulata, Good.)

var. conferta, (Chapm.), Bailey.

var. radiata, (Wahl.) (C. stellulata, Good., vars. scirpoides and angustata, Carey, and $C$. sterilis, Willd.)

Emmonsii, Dewey.

exilis, Dewey.

extensa, Good.

filiformis, $\mathrm{L}$.

var. lanuginosa, (Michx.) (C. lanuginosa, Michx.)

flava, $\mathrm{L}$.

flexilis, Rudge.

folliculata, L.

formosa, Dewey.

glaucodea, Tuck. (C. flaccosperma, Gray, not Devey.)

gracillima, Schw.

granularis, Mubl.

var. Haleana, Porter.

Grayii, Carey.

grisea, Wahl.

Hitchcockiana, Dewey.

hystricina, Muhl.

intumescens, Rudge.

laxiflora, Iam.

var. blanda, (Dewey), Boott.

var. heterosperma, (Wahl.) (var. intermeclia, Boott.)

var. latifolia, Boott.

var. plantaginea, (Schk.), Boott.

var. styloflexa, (Buckley), Boott.

limosa, L.

livida, Willd.

longirostris, Torr.

lurida, Wahl. (C. lupulina, Muhl.)

var. polystachya, (Schw. \& Torr.), Bailey. (C. lupuliformis, Sartw.)

Magellanica, Lam., var. irrigua, (Smith). (C. irrigua, Smith.) monile, Tuck.

Muhlenbergii, Schk.

var. enervis, Boott.

nigro-marginata, Schw.

Novæ-Angliæ, Schw.

Ederi, Retz. 
oligocarpa, Schk.

oligosperma, Michx.

pallescens, $\mathbf{L}$.

pauciflora, Lightf.

pedunculata, Muhl.

Pennsylvanica, Lam.

plantaginea, Lam.

platyphylla, Carey.

polymorpha, Muhl.

polytrichoides, Muhl.

prasina, Wahl. (C. miliacea, Muhl.)

Pseudo-Cyperus, L.

var. comosa, (Boott), W. Boott.

ptychocarpa, Steud.

pubescens, Muhl.

retrocurva, Dewey.

retrorsa, Schw.

riparia, Curtis.

rosea, Schk.

var. radiata, Dewey. (Includes var. minor, Boott.)

var. retroflexa, (Muhl.), Torr. (C. retroflexa, Muhl.)

rostrata, With.

var. utriculata, (Boott), Bailey. (C. utriculata, Boott.)

salina, Wahl. (Monmouth Co.?)

scabrata, Schw.

Schweinitzii, Dewey.

scirpoidea, Michx.

scoparia, Schk.

siccata, Dewey.

Smithii, Porter.

sparganioides, Muhl.

squarrosa, L.

stipata, Muhl.

straminea, Schk. (Includes vars. typica, tenera, Crawei and festucacea, Boott.)

var. alata, (Torr.), Bailey. (C. alata, Torr.)

var. fœnea, (Willd.), Torr. (C. foenea, Willd.)

var. mirabilis, (Dewey), Tuck. (C. cristata. schw., var. mirce

bilis, Gray, in part.)

var. moniliformis, Tuck. (C. foenea, Willd., var.(.) sallu. lonum, Gray.)

striata, Michx. 
stricta, Lam.

subulata, Michx.

Sullivantii, Boott.

tenella, Schk.

tentaculata, Muhl.

var. gracilis, Boott.

teretiuscula, Good. (Inclucles var. major, Koch.)

tetanica, Schk.

var. Canbyi, (Olney), Porter. (C. panicca, I., var. Canbyi, Olney.)

torta, Boott.

tribuloides, Wahl. (C. lagopoctioides, Schk.)

var. cristata, (Schw.), Bailey. (C. cristuta, Schw.)

triceps, Michx.

trichocarpa, Mubl.

trisperma, Dewey.

Tuckermani, Boott.

umbellata, Schk.

varia, Muhl.

venusta, Dewey, var. glabra, (Boott), Bailey. (C. glabra, Boott.)

vestita, Willd.

virescens, Muhl.

vulgaris, Fries.

vulpinoidea, Michx.

Willdenovii, Schk.

\section{Paspalum, $\mathbf{L}$.}

GRAMINE正.

læve, Michx.

setaceum, Michx.

Walterianum, Schult.

Amphicarpum, Kunth.

Purshii, Kunth.

\section{Panicum, $\mathbf{L}$.}

agrostoides, Muhl.

amarum, Ell., var. minor, V. \& S.

anceps, Michx.

capillare, L.

clandestinum, L.

crus-galli, L.

var. hispidum, (Mubl.), Torr.

depauperatum, Mubl.

dichotomum, L. 

var. barbulatum, (Michx.), Gray.

discolor, (Chapm.), Steud. ?

filiforme, $\mathrm{L}$.

glalirum, (Schrad.), Gaud.

latifolium, L.

var. molle, Vasey.

laxiflorum, Lam.

microcarpon, Muhl.

miliaceum, $L$.

nitidum, Lam.

proliferum, Lam.

sanguinale, $L$.

scoparium, Lam. (P. pauciflorum, Ell.?)

verrucosum, Muhl.

virgatum, $\mathrm{L}$.

viscidum, Ell.

xanthophysum, Gray.

Setaria, Beauv.

glauca, (L.), Beauv.

Italica, $(L)$, Kunth.

verticillata, $(L$.$) , Beauv.$

viridis, $(L$.), Beauv.

Cenchrus, $\mathbf{I}$.

tribuloides, L.

Spartina, Schreb.

cynosuroides, (L.), Willd.

juncea, (Michx.), Willd.

polystachya, (Michx.), Willd.

stricta, (Ait.), Roth.

var. maritima, (Walt.) (var. alterniflora, Gray.)

var. glabra, (Muh].), Gray.

'Tripsacum, L.

dactyloides, $\mathrm{L}$.

Zizania, $\mathrm{L}$.

aquatica, $\mathrm{L}$.

Ieersia, Sw.

oryzoides, (L.), Sw.

Virginica, Willd.

Erianthus, Michx.

alopecuroides, (L.), Ell.

Andropogon, L.

dissitiflorus, Michx. (A. Virginicus, Gray, not $L_{\text {. }}$ ) 

glomeratus, (Walt.) (A. macrourus, Michx.)

provincialis, Lam. (A. furcatus, Muhl.)

var. maritimus, (Chapm.), Hack.

scoparius, Michx.

Clurysopogon, Trin.

nutans, (L.), Benth. (Sorghum nutans, Gray.)

Phalaris, L.

arundinacea, $L$.

Canariensis, $L$.

Anthoxanthum, L.

odoratum, $I$.

Hierochloa, Gnel.

odorata, (L.) (H. borealis, Roem. \& Schult.)

Alopecurus, $\mathbf{L}$.

geniculatus, $I$.

var. aristulatus, (Michx.), Munro.

Aristida, L.

dichotoma, L.

gracilis, Ell.

purpurascens, Poir.

tuberculosa, Nutt.

Stipa, L.

avenacea, $\mathrm{L}$.

Oryzopsis, Michx.

. asperifolia, Michx.

juncea, (Michx.) (O. Canadensis, T'orr.)

melanocarpa, Muhl.

Milium, L.

effusum, $\mathrm{L}$.

Muhlenbergia, Schreb.

capillaris, Kunth.

diffusa, Schreb.

Mexicana, (L.), Trin.

racemosa, (Michx.) (M. glomerata, Trin.)a sobolifera, (Muhl.), Trin.

sylvatica, (Torr.), T. \& G.

tenuiflora, (Willd.) (M. Willdenovii, Trin.)

Brachyelytrum, Beauv.

aristosum, (Michx.) (B. aristatum, Beauv.)

Heleochloa, Host.

schoenoides, (L.), Host. (Crypsis schcenoides, Lam.) 
,

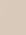


Phleum, L.

pratense, $L$.

Sporobolus, R. Br.

asper, (Michx.), Kunth. (Vilfa aspera, Beauv.)

compressus, (Torr.), Kunth.

heterolepis, Gray.

serotinus, (Torr.), Gray.

vaginæflorus, (Torr.), Vasey. (V. vaginceflora, T'orr.)

Agrostis, L.

alba, $L$.

var.vulgaris, (With.), Thurb. (A.vulgaris, With)

altissima, (Walt.), Tuck. (A. elata, Trin.)

canina, L.

hiemalis, (Walt.) (A. scabra, Willd.)

perennans, (Walt.), Tuck.

Polypogon, Desf.

Monspeliensis, (L.), Desf.

Cinna, L.

arundinacea, $\mathrm{L}$.

pendula, Trin. (C. arundinacea, L., var. pendula, Gray.)

Deyeuxia, Clarion.

Canadensis, (Michx.), Beauv. (Calamagrostis Canadensis, Beauv.)

Nuttalliana, (Steud.), Vasey. (C. Nuttalliana, Steud.)

Ammophila, Host.

arenaria, (L.), Link. (Calamagrostis arenaria, Roth.) brevipilis, (Torr.), Benth. (C. brevipilis, Gray.)

Aira, L.

caryophyllea, $L$.

proecox, $L$.

Deschampsia, Beauv.

cæspitosa, (L.), Beauv.

flexuosa, (L.), Griseb.

Holcus, $\mathbf{L}$.

lanatus, $L$.

Trisetum, Pers.

Pennsylvanicum, (L.) (T. palustre, Torr.)

Avena, $\mathbf{L}$.

striata, Michx.

Arrhenatherum, Beauv.

elatius, (L.), Mert. \& Koch. (A. avenaceum, Beauv.)

Danthonia, DC.

Alleni, Austin. 
compressa, Austin.

sericea, Nutt.

spicata, (L.), Beauv.

Cynorlon, Pers.

Dactylon, (L.), Pers.

Gymmopogon, Beauv.

ambiguus, (Michx.) (G.racemosus, Bcauv.)

Bouteloua, Lag.

curtipendula, (Michx.', Gray.

Eleusine, Grertn.

Indica, $\left(L_{0}\right)$, Gcertn.

Triodia, R. Br.

sesleroides, (Michx.), Benth. ('Tricuspis sesleroides, 'T'or r.)

Diplachne, Beauv.

fascicularis, (Lam.), Beauv. (Leptochloa fascicularis, Gray.)

Triplasis, Beauv.

purpurea, (Walt.), Chapm. (Tricuspis purpurea, Gray.)

Phragmites, Trin.

vulgaris, (Lam.) (P. communis, T'rin.)

Cynosurus, L.

cristatus, $L$.

Eatonia, Raf.

Dudleyi, Vasey.

obtusata, (Michx.), Gray.

Pennsylvanica, (Spreng.), Gray.

Eragrostis, Beauv.

capillaris, (L.), Nees.

Frankii, Meyer.

hypnoides, (Lam.) (E. reptans, Nees.)

major, Host. (E. poceoides, Beauv., var. megastachya, Gray.)

minor, Host. (E. pocoides, Beauv.)

pectinacea, (Michx.), Gray.

var. spectabilis, (Pursh), Gray.

pilosa, (L.), Beauv.

Purshii, Schrad.

\section{Uniola, $\mathbf{L}$.}

laxa, (L.) (U. gracilis, Michx.)

\section{Distichlis, Raf.}

spicata, (L.), Greene. (Brizopyrum spicatum, Hook.)

Dactylis, $\mathbf{L}$.

glomerata, $L$.

Briza, $\mathbf{L}$.

media, $L$. 


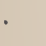


Poa, I.

alsodes, Gray.

annua, $\mathrm{L}$.

autumnalis, Muhl. (P. flexuosa, Muhl.)

compressa, $\mathrm{L}$.

debilis, Torr.

pratensis, $\mathrm{L}$.

serotina, Ehrh.

sylvestris, Gray.

trivialis, $L$.

Glyceria, R. Br.

arundinacea, Kunth. (G. aquatica, Gray, Manual.) brevifolia, (Muhl.), Schult. (G. acutiflora, Torr.)

Canadensis, (Michx.), Trin.

distans, (L.), Wah].

elongata, (Torr.), Trin.

fluitans, (L.), R. Br.

maritima, (Huds.), Mert. \& Kíoch.

nervata, (Willd.), Trin.

obtusa, (Nutt.), Trin.

pallida, (Eddy), Trin.

Festuca, I.

duriuscula, L. (F. ovina, L., var. duriuscula, Gray.)

elatior, $L$.

gigantea, (L.), Vill.

Myurus, $L$.

nutans, Spreng.

octoflora, Walt. (F. tenella, Willd.)

ovina, $L$.

rubra, L. (F. ovina, L., var. rubra, Gray.)

\section{Bromus, I.}

ciliatus, $\mathrm{L}$.

Kalmii, Gray.

mollis, $L$.

purgans, L. (B. ciliatus, L., var. purgans, Gray.)

racemosus, $I$.

secalinus, $I$.

sterilis, $I$.

tectorum, $L$.

Lolium, I.

perenne, $L$. 
Agropyrum, J. Gærtn.

caninum, (L.), Rœm. \& Schult. ('I'riticum caninum, Gray, Manual.)

repens, (L.), Beauv. (T. repens, L.)

Hordeum, L.

jubatum, $L$.

Elymus, L.

Canadensis, L.

var. glaucifolius, (Mubl.), Gray.

striatus, Willd.

Virginicus, L.

Asprella, Willd.

Hystrix, (L.), Willd. (Gymnostichum Hystrix, Schreb.)

Thuja, L.

CONIFER $\mathrm{E}$.

occidentalis, $\mathrm{L}$.

Chamacyparis, Spach.

thyoides, (L.) (Cupressus thyoides, L.)

Juniperus, L.

communis, L.

Virginiana, L.

Taxus, L.

baccata, L., var. Canadensis, (Willd.), Gray.

Pinus, $\mathbf{L}$.

echinata, Mill. (P. mitis, Michx.)

inops, Ait.

pungens, Michx. f.

resinosa, Ait.

rigida, Mill.

Strobus, L.

Picen, Link.

Canadensis, (Mill.) (Abies alba, Michx.)

Mariana, (Mill.) (A.nigra, Poir.)

Tsuga, Carr.

Canadensis, (L.), Carr. (Abies Canadensis, Michx.)

Abies, Juss.

balsamea, (L.), Mill.

Larix, Mill.

laricina, (DuRoi). (L. Americana, Michx.) 

Isoetes, L.

ISOETE正。

echinospora, Durieu, var. Braunii, (Durieu), Engelm.

Engelmanni, A. Br.

lacustris, $\mathrm{L}$.

riparia, Engelm.

Selaginella, Beaur.

SELAGINELLEÆ.

apus, (L.), Spring.

rupestris, (L.), Spring.

Marsilia, L.

RHIZOCARP正。

quadrifolia, $L$.

Azolla, Lam.

Caroliniana, Willd.

Lycopodium, $\mathbf{L}$.

LYCOPODIACE正.

alopecuroides, $\mathrm{L}$.

annotinum, L.

Carolinianum, $\mathrm{L}$.

clavatum, L.

complanatum, L.

inundatum, $\mathrm{L}$.

lucidulum, Michx.

obscurum, L. (L. dendroideum, Michx.)

Selago, L.

Ophioglossum, L.

OPHIOGLOSSACE正.

vulgatum, L.

Botrychium, Sw.

lanceolatum, (Gmel.), Angstr.

matricariæfolium, A. Br.

simplex, Hitchcock.

ternatum, (Thunb.), Sw.

var. dissectum, (Spreng.), Milde.

var. intermedium, D. C. Eaton. (B. lunarioides, D. C. Eaton, in Gray, Manual.)

var. obliquum, (Muhl.), Milde.

Virginianum, (L.), Sw.

Polypodium, $\mathbf{L}$.

FILIC்ES.

vulgare, $\mathbf{L}$. 

Cheilanthes, Sw. vestita, (Spreng.), Sw.

Pellaea, Iink. atropurpurea, (L.), Link. gracilis, (Michx.), Hook.

Pteris, I. aquilina, L. var. caudata, (L.), Hook.

\section{Adiantum, $\mathbf{L}$.} pedatum, L.

Woodwardia, Smith. areolata, (L.), Moore. (W. angustifolia, Smith) Virginica, (L.), Smith.

\section{Asplenium, $\mathbf{L}$.}

acrostichoides, Sw. (A. thelyptervides, Michx.)

angustifolium, Michx.

Bradleyi, D. C. Eaton.

ebenoides, R. R. Scott.

Filix-fœmina, (L.). Bernh.

var. laciniatum. Moore.

montanum, Willd.

pinnatifidum, (Mubl.), Nutt.

platyneuron, (L.) (A. ebeneum, Ait.)

var. serratum, (E. S. Miller).

Ruta-muraria, $\mathrm{L}$

Trichomanes, L.

Camptosorus, Link.

rhizophyllus, (L.), Link.

\section{Phegopteris, Fée.}

connectilis, (Michx.) (P.polypodioides, Fée.)

Dryopteris, (L.), Fée.

hexagonoptera, (L.), Fée.

\section{Aspidium, Sw.}

acrostichoides, (Michx.), Sw.

var. Schweinitzii, (Beck.) (var. incisum, Gray.)

aculeatum, Sw., var. Braunii, (Spenner), Doell.

Boottii, Tuck. (A. spinulosum, Sw., var. Boottii, Gray.)

cristatum, (L.), Sw.

var. Clintonianum, D. C. Eaton.

Goldianum, Hook.

marginale, (L.), Sw.

Noveboracense, (L.), Sw. 


$$
\text { . }
$$


spinulosum, Sw.

var. dilatatum, (Sw.), Hook.

var. intermedium, (Willd.), D. C. Eaton.

Thelypteris, (L.), Sw.

Cystopteris, Bernh.

bulbifera, (L.), Bernh.

fragilis, (L.), Sw.

Onoclea, $\mathrm{L}$.

sensibilis, $\mathrm{L}$.

Struthiopteris, (L.), Hoffm. (Struthiopteris Germanica, Willd.)

Woodsia, R. Br.

Ilvensis, (L.), R. Br.

obtusa, (Spreng.), Torr.

Dicksonia, L'Hér.

punctilobula, (Michx.), Gray.

Lygodium, Sw.

palmatum, (Bernh.), Sw.

Schizæa, Smith.

tortuosa, Pursh. (S. pusilla, Pursh.)

Osmunda, $\mathbf{L}$.

cinnamomea, $\mathrm{L}$.

Claytoniana, $\mathbf{L}$.

regalis, $\mathbf{L}$.

EQUISETACE ÆE.

Equisetum, I.

arvense, L.

var. serotinum, Meyer.

hiemale, L.

limosum, $\mathrm{L}$.

littorale, Kuhlw. ?

sylvaticum, L. 


\section{BALLAST PLANTS}

Found in the Vicinity of New York and Philadelphia, and mainly collected and identified by Hon. Addison Brown, I. C. Martindale, C. F. Parker, C. E. Smith and Isaac Burk.

\section{RANUNCULACE $\nexists$.}

Ranunculus arvensis, L.

Ranunculus Græcus, Griseb.

Ranunculus lanuginosus, $\mathrm{L}$.

Ranunculus parvulus, L.

Ranunculus Sardous, Crantz.

Ranunculus villosus, DC.

Delphinium Ajacis, Reichenb.

Delphinium divaricatum, Ledeb.

Delphinium peregrinum, L.

\section{PAPAVERACE $\AA$.}

Papaver hybridum, L.

Papaver Rhœas, $\mathbf{L}$.

Eschscholtzia Californica, Cham.

Hypecoum grandiflorum, Benth.

Fumaria capreolata, L.

Fumaria parviflora, Lam.

\section{CRUCIFER丑.}

Nasturtium officinale, R. Br., var. microphyllum, Reichenb.

Alyssum incanum, R. Br.

Sisymbrium Irio, L.

Sisymbrium Lœeselii, L.

Sisymbrium Pannonicum, Jacq.

Sisymbrium polyceratium, L.

Erysimum orientale, $\mathrm{L}$.

Erysimum repandum, L.

Brassica Cheiranthus, Vill.

Brassica Monensis, Huds.

Brassica Napus, L.

Brassica oleracea, L.

Diplotaxis erucoides, DC. 
Diplotaxis muralis, DC.

Diplotaxis tenuifolia, DC.

Diplotaxis virgata, DC.

Lepidium graminifolium, L.

Lepidium latifolium, L.

Lepidium perfoliatum, L

Lepidium sativum, L.

Lepidium Smithii, Hook.

Iberis umbellata, $\mathrm{L}$.

Teesdalia nudicaulis, R. Br.

Neslia paniculata, Desv.

Bunias orientalis, L.

Rapistrum rugosum, All.

Cakile maritima, Scop.

Raphanus maritimus, Smith.

Polanisia viscosa, DC.

CAPPARIDE㘴.

RESEDACE正.

Reseda alba, L.

Reseda lutea, L.

Reseda odorata, L.

Reseda Phyteuma, J.

VIOLARIE 刃E.

Ionidium parviflorum, Vent.

FRANKENIACE $巴$.

Frankenia pulverulenta, $\mathrm{L}$.

CARYOPHYLLEÆ.

Dianthus barbatus, L.

Gypsophila muralis, L.

Saponaria ocymoides, L.

Silene Anglica, L.

Silene conoidea, L.

Silene dichotoma, Ehrh.

Silene Gallica, L.

Silene Italica, Pers.

Silene pendula, L.

Silene saponariæfolia, L.

Lychnis Chalcedonica, L.

Lychnis Flos-cuculi, L. 
Lychnis sylvestris, Schk.

Cerastium tetrandrum, Curtis.

Stellaria aquatica, Scop.

Sagina maritima, Smith.

Polycarpon tetraphyllum, L.

PORTULACE无.

Portulaca pilosa, L.

Portulaca splendens, L.

HYPERICINE正。

Hypericum humifusum, L.

MALVACE无.

Althæa hirsuta, L.

Malva arvensis, Presl.

Malva borealis, Wallm.

Malva Nicæensis, All.

Malva parviflora, L.

Malvastrum spicatum, Gray.

Malvastrum tricuspidatum, Gray.

Sida angustifolia, Juss.

Sida carpinifolia, L.

Sida carpinifolia, L., var. brevicuspidata, Griseb.

Sida cordifolia, L.

Sida linifolia, Cav.

Sida rhombifolia, L.

Abutilon cordifolium, L.

Sphæralcea miniata, Cav.

Modiola multifida, Mœench.

Pavonia hastata, Cav.

Urena lobata, L.

Gossypium album, Ham.

Gossypium Barbadense, L.

STERCULIACEA.

Waltheria Americana, L.

TILIACE 2 .

Corchorus fascicularis, Lam.

Corchorus tridens, $\mathrm{L}$.

Corchorus trilocularis, $\mathbf{L}$.

Linum angustifolium, Huds.

LINEA. 
ZYGOPHYLLE压.

Tribulus terrestris, $\mathrm{L}$.

Zygophyllum Fabago, L.

GERANIACEAE.

Geranium molle, L.

Geranium rotundifolium, L.

Erodium Ciconium, Willd.

Erodium gruinum, Willd.

Erodium malachoides, Willd.

Erodium moschatum, L'Hér.

SAPINDACE $\nexists$.

Cardiospermum Halicacabum, L.

LEGUMINOSAE.

Lupinus angustifolius, L.

Genista scoparia, Lam.

Ulex nanus, Forst.

Ononis antiquorum, $\mathrm{L}$.

Ononis arvensis, $\mathrm{L}$.

Ononis repens, $\mathrm{L}$.

Ononis spinosa, L.

Trigonella Besseriana, L.

Trigonella cærulea, Ser.

Trigonella corniculata, L.

Trigonella Monspeliaca, L.

Trigonella ornithopoides, DC.

Trigonella polycerata, L.

Medicago apiculata, Willd.

Medicago ciliaris, Willd.

Medicago denticulata, Willd.

Medicago Echinus, Willd.

Medicago falcata, L.

Medicago flexuosa, Ten.

Medicago lappacea, Desr.

Medicago littoralis, Rohdl.

Medicago marginata, Willd.

Medicago minima, $\mathrm{L}$.

Medicago muricata, All.

Medicago orbicularis, L.

Medicago pubescens, DC.

Medicago spinulosa, DC. 
Medicago tribuloides, L.

Medicago turbinata, Willd.

Melilotus arvensis, Wallr.

Melilotus compacta, Salz.

Melilotus gracilis, DC.

Melilotus infesta, Guss.

Melilotus macrorhiza, Pers.

Melilotus messanensis, Desf.

Melilotus occidentalis, Nutt.

Melilotus parviflora, Desf.

Melilotus sulcata, Desf.

Trifolium angustifolium, L.

Trifolium Dalmaticum, Vis.

Trifolium elegans, Savi.

Trifolium fragiferum, L.

Trifolium lappaceum, L.

Trifolium maritimum, Huds.

Trifolium ochroleucum, L.

Trifolium resupinatum, $\mathrm{L}$.

Trifolium scabrum, L.

Trifolium striatum, L.

Trifolium subterraneum, L.

Trifolium tomentosum, L.

Anthyllis vulneraria, L.

Dorycnium hirsutum, DC.

Lotus angustissimus, L.

Lotus corniculatus, L.

Lotus tenuifolius, Presl.

Psoralea bituminosa, L.

Sesbania Floridana, S. Wats.

Sesbania macrocarpa, Muhl.

Colutea arborescens, L.

Astragalus glycyphyllos, L.

Scorpiurus subvillosa, L.

Scorpiurus vermiculata, I.

Ornithopus compressus, L.

Ornithopus perpusillus, L.

Ornithopus scorpioides, L.

Coronilla Emerus, L.

Hippocrepis comosa, L.

Adesmia muricata, DC.

var. dentata, (DC.) 
Arachis hypogæa, Willd.

Vicia calcarata, Desf.

Vicia grandiflora, Scop.

Vicia hybrida, L.

Vicia lutea, L.

Vicia micrantha, Nutt.

Vicia Narbonensis, L.

Vicia peregrina, $\mathrm{L}$.

Ervum Lens, L.

Lathyrus Aphaca, L.

Lathyrus ochrus, DC.

Lathyrus sativus, $\mathrm{L}$.

Pisum sativum, L.

Vigna glabra, Savi.

Vigna luteola, Benth.

Rhynchosia minima, DC.

Cassia occidentalis, L.

Desmanthus brachylobus, Benth.

ROSACE无.

Rubus fruticosus, L.

Rubus glandulosus, Bell.

Potentilla reptans, L.

Alchemilla arvensis, Scop.

Epilobium hirsutum, L.

ONAGRARIE无.

Epilobium parviflorum, Schreb.

Epilobium pubescens, Roth.

Jussiæa leptocarpa, Nutt.

Gaura sinuata, Nutt.

CUCURBITACEAE.

Ecbalium agreste, Rich.

FICOIDE正.

Mesembryanthemum crystallinum, L.

Mesembryanthemum nodosum, $\mathrm{L}$.

Trianthema monogynum, L.

Mollugo Cerviana, Ser.

UMBELLIFER丑.

Eryngium campestre, L.

Eryngium maritimum, L. 
$-$

- 
Bupleurum protractum, Link.

Apium divaricatum, S. Wats.

Apium graveolens, L.

Apium leptophyllum, F. Muell.

Apium Petroselinum, L.

Apium repens, Reichenb.

Ammi majus, L.

Ammi Visnaga, L.

Chierophyllum sylvestre, L.

Chærophyllum temulum, L.

Scandix Pecten-Veneris, L.

Enanthe Phellandrium, Lam.

Peucedanum graveolens, S. Wats.

Heracleum Spondylium, I.

Coriandrum sativum, L.

Bifora radians, Bieb.

Daucus Broteri, Ten.

Caucalis Helvetica, Jacq.

Caucalis infesta, Curtis.

Caucalis latifolia, L.

Caucalis nodosa, Scop.

CAPRIFOLIACEZ.

Sambucus Ebulus, L.

RUBIACE无。

Richardsonia scabra, St. Hil.

Galium palustre, L.

Galium Parisiense, L.

Galium tricorne, With.

Asperula arvensis, L.

Sherardia arvensis, L.

VALERIANEAE.

Valerianella carinata, Loisel.

Valerianella dentata, Koch.

Valerianella Morisonii, DC.

DIPSACEAE.

Scabiosa stellata, L.

CALYCERE无.

Calycora balsamitefolia, Rich. 



\section{COMPOSIT无.}

Ageratum conyzoides, L.

Eupatorium cannabinum, L.

Mikania gonoclada, DC.

Grindelia glutinosa, Dun.

Heterotheca subaxillaris, (Lam.), Britton \& Rusby.

Solidago tortifolia, Ell.

Bellis perennis, $\mathrm{L}$.

Aster oblongifolius, Nutt.

Aster Tripolium, L.

Erigeron acris, L.

Erigeron divaricatus, Michx.

Erigeron linifolius, Willd.

Conyza albida, Less.

Baccharis Douglasii, DC.

Filago arvensis, L.

Filago minima, Fries.

Gnaphalium luteo-album, L.

Gnaphalium palustre, Nutt.

Inula dysenterica, L.

Inula Pulicaria, L.

Inula viscosa, Desf.

Buphthalmum salicifolium, L.

Acanthospermum hispidum, DC.

Acanthospermum humile, DC.

Acanthospermum xanthioides, DC.

Parthenium Hysterophorus, L.

Ambrosia psilostachya, DC.

Heliopsis buphthalmoides, Dun.

Helianthus petiolaris, Nutt.

Melanthera deltoidea, Michx.

Spilanthes repens, Michx.

Guizotia oleifera, DC.

Bidens leucantha, Willd.

Bidens tripartita, L.

Hemizonia fasciculata, T. \& G., var. ramosissima, Gray.

Hemizonia pungens, T. \& G.

Flaveria Contrayerba, Pers.

Helenium quadridentatum, Labill.

Anacyclus tomentosus, $\mathrm{L}$.

Achillea Ptarmica, L.

Achillea rosea, Desf. 


$$
\text { - }
$$


Anthemis mixta, L.

Anthemis secundiramea, Biron.

Chrysanthemum coronarium, L.

Chrysanthemum segetum, L.

Matricaria discoidea, DC.

Matricaria maritima, I.

Artemisia Dracunculus, L.

Cenia turbinata, Pers.

Senecio chrysanthemifolius, Poir.

Senecio erucæfolius, L.

Senecio Jacobæus, L.

Senecio sylvaticus, L.

Senecio viscosus, L.

Calendula arvensis, L.

Calendula officinalis, I.

Carduus acanthoides, L.

Carduus crispus. I.

Carduus Marianus, L.

Carduus multiflorus, Gaud.

Carduus pycnocephalus, L.

Cnicus arvensis, Hoffm., var. integrifolia, (Koch.)

Silybum Marianum, Gærtn.

Serratula tinctoria, L.

Centaurea aspera, L.

Centaurea Austriaca, Willd.

Centaurea Jacea, I.

Centaurea paniculata, L.

Centaurea Phrygia, L.

Centaurea Scabiosa, L.

Centaurea solstitialis, L.

Centaurea tenuifolia, Dufour.

Carthamus lanatus, I.

Scolymus Hispanicus, L.

Cichorium divaricatum, Schreb.

Cichorium Endivia, L.

Arnoseris pusilla, Gærtn.

Picris echioides, $\mathrm{L}$.

Picris hieracioides, $\mathrm{L}$.

Picris hispida, All.

Hieracium prenanthoides, Vill.

Hieracium umbellatum, L.

Hypochæris glabra, L. 

Hypochæris radicata, L.

Leontodon hirtus, L.

Leontodon hispidus, L.

Leontodon pratensis, Koch.

Sonchus tenerrimus, L.

Jasione montana, L.

Wahlenbergia linarioides, DC.

Campanula urticæfolia, Schmid.

Specularia hybrida, DC.

Specularia Speculum, DC.

PLUMBAGINE正.

Armeria maritima, Willd.

PRIMULACEAE.

Anagallis cærulea, Smith.

ASCLEPIADEÆ.

Philibertia gracilis, D. Don.

POLEMONIACE $Æ$.

Gilia achilleæfolia, Benth.

Gilia capitata, Dougl.

HYDROPHYLLACE无.

Nama Jamaicense, L.

BORRAGINEZE.

Tournefortia heliotropioides, Hook.

Heliotropium anchusæfolium, Poir.

Heliotropium Curassavicum, L.

Heliotropium Indicum, L.

Heliotropium Peruvianum, Don.

Heliotropium supinum, L.

Echinospermum Redowskii, Lehm.

Amsinckia intermedia, Fisch \& Mey.

Amsinckia supina, L.

Borrago officinalis, L.

Anchusa leptophylla, Rœm. \& Schult.

Anchusa officinalis, Don.

Nonnea nigricans, DC.

Myosotis collina, Hoffm.

Myosotis versicolor, Pers.

Echium pustulatum, Sibth.

Echium violaceum, L. 
CONVOLVULACE $\nexists$.

Ipomœa acetosæfolia, Vahl.

Ipomœa commutata, Rœm. \& Schult.

Ipomøa hederacea, Jacq., var. integriuscula, Gray.

Ipomœa lacunosa, L.

Ipomœa littoralis, Choisy.

Ipomœa sinuata, Ort.

Ipomœa tamnifolia, L.

Convolvulus pentapetaloides, L.

Convolvulus tricolor, L.

Dichondra repens, Forst.

SOLANACERE.

Solanum Balbisii, Dun.

Solanum elæagnifolium, Cav.

Solanum gracile, Link.

Solanum mammosum, L.

Solanum miniatum, Bernh.

Solanum nigrum, L., var. Dillenii, Gray.

Solanum nigrum, L., var. nudiflorum, Gray.

Solanum nigrum, L., var. villosum, Mill.

Solanum sisymbriifolium, Lam.

Physalis Peruviana, Nees.

Atropa Belladonna, L.

Datura Metel, L.

Hyoscyamus albus, L.

Nicotiana glauca, Grah.

Nicotiana longiflora, Cav.

Petunia parviflora, Juss.

Petunia violacea, Hook.

SCROPHULARINE无.

Verbascum nigrum, L.

Verbascum orientale, Bieb.

Verbascum phlomoides, L.

Verbascum pulverulentum, Vill.

Verbascum ramigerum, Link.

Verbascum sinuatum, L.

Verbascum virgatum, With.

Linaria Cymbalaria, Mill.

Linaria minor, Desf.

Linaria repens, Steud.

Linaria spuria, Mill. 
Linaria striata, DC.

Linaria supina, Desf.

Antirrhinum majus, L.

Antirrhinum Orontium, L.

Scrophularia aquatica, L.

Scrophularia canina, L.

Scrophularia Hoppei, Koch.

Herpestis Monniera, HBK.

Scoparia flava, Cham. \& Sch.

Digitalis lutea, L.

Veronica Beccabunga, L.

Veronica Cymbalaria, Bertol.

OROBANCHACE王.

Orobanche minor, L., var. flavescens, DC.

Sesamum Indicum, L.

PEDALINE王.

VERBENACEA.

Lippia nodiflora, Michx.

Verbena Bonariensis, L.

Verbena littoralis, HBK.

LABIAT AE.

Mentha Pulegium, L.

Satureia montana, L.

Calamintha officinalis, Mœench.

Salvia Verbenaca, L.

Brunella laciniata, $\mathrm{L}$.

Stachys annua, L.

Stachys hirta, L.

Stachys recta, L.

Stachys sylvatica, L.

Galeopsis angustifolia, Ehrh.

Galeopsis versicolor, Curtis.

Galeopsis villosa, Huds.

Leonurus glaucescens, Bunge.

Teucrium Scordium, L.

Ajuga Chamrepitys, Schreb.

Ajuga Genevensis, L.

Plantago Coronopus, L.

PLANTAGINE $\not$.

Plantago Lagopus, L.

Plantago heterophylla, Nutt.

Plantago hirtella, HBK. 


\section{ILLECEBRACE AE.}

Corrigiola littoralis, L.

Herniaria glabra, L.

Herniaria hirsuta, L.

Celosia cristata, L.

AMARANTACEAE.

Amarantus blitoides, S. Wats.

Amarantus crispus, L.

Amarantus deflexus, L.

Amarantus polygonoides, L.

Amarantus sanguineus, $\mathrm{L}$.

Alternanthera Achyrantha, R. Br.

Cladothrix lanuginosa, Moq.

Gomphrena globosa, L.

\section{CHENOPODIACEAE.}

Chenopodium obovatum, DC.

Chenopodium polyspermum, L.

Chenopodium Vulvaria, L.

Beta maritima, L.

Beta trigyna, L.

Atriplex congesta, Moq.

Atriplex hortensis, L.

Atriplex laciniata, $\mathrm{L}$.

Polycnemon arvensis, L.

Kochia scoparia, Schrad.

POLYGONACEAE.

Polygonum hernarioides, Del.

Polygonum lapathifolium, L.

var. incanum, Gray.

Polygonum minus, Huds.

Rumex aquaticus, L.

Rumex conglomeratus, Miers.

Rumex pulcher, L.

Rumex sanguineus, $\mathrm{L}$.

Emex spinosa, Camb.

EUPHORBIACE AE.

Euphorbia Esula, L.

Euphorbia exigua, L.

Euphorbia hiberna, L.

Euphorbia humistrata, Engelm. 
Euphorbia Peplis, L.

Euphorbia Peplus, L.

Euphorbia platyphylla, L.

Euphorbia segetalis, L.

Euphorbia serpens, HBK.

Phyllanthus polygonoides, Spreng.

Croton glandulosus, L.

Croton maritimus, Walt.

Mercurialis annua, L.

Acalypha Poiretii, Spreng.

Ricinus communis, L.

URTICACE E

Parietaria debilis, Forst.

Parietaria diffusa, Mert. \& Koch.

Parietaria officinalis, L.

IRIDE $A$.

Iris Pseudacorus, L.

LILIACEA.

Asphodelus ramosus, L.

CYPERACEA

Cyperus echinatus, (EII.), Britton.

Cyperus flavo-mariscus, Griseb.

Cyperus fuscus, $L$.

Cyperus Lecontei, Torr.

Cyperus Surinamensis, Rottb.

Cyperus umbellatus, Vahl.

Fimbristylis miliacea, Vahl.

Scirpus affinis, Roth.

Scirpus glaucus, L.

Scirpus Holoschœnus, L.

Scirpus setaceus, L.

Carex Davalliana, Lam.

Carex distans, L.

Carex hirta, L.

Carex muricata, L.

Carex ornithopoda, With.

GRAMINE A.

Paspalum ciliatifolium, Michx.

Paspalum distichum, $L$.

Paspalum notatum, Fluegg.

l'aspalum plicatulum, Michx. 
Paspalum purpurascens, Ell.

Paspalum scrobiculatum, $\mathbf{L}$.

Paspalum undulatum, Poir.

Paspalum vaginatum, $\mathrm{S} w$.

Panicum colonum, L.

Panicum prostratum, Lam.

Setaria composita, HBK.

Setaria setosa, Beauv.

Tragus racemosus, Hall.

Andropogon Halapensis, Sibth.

Andropogon Ischæmon, L.

Phalaris intermedia, Bosc.

Phalaris paradoxa, $\mathrm{L}$.

Hierochloa australis, Rœm. \& Schult.

Alopecurus agrestis, L.

Alopecurus bulbosus, L.

Alopecurus Creticus, Trin.

Phleum arenarium, $\mathrm{L}$.

Phleum asperum, Vill.

Phleum Bohmeri, Wibel.

Phleum tenue, Schrad.

Sporobolus cryptandrus, Gray.

Sporobolus Indicus, R. Br.

Agrostis verticillata, Vill.

Apera Spica-venti, Beauv.

Corynephorus canescens, Beauv.

Holcus mollis, L.

Arena fatua, L.

Gaudinia fragilis, Beauv.

Chloris petræa, $\mathrm{Sw}$.

Eleusine ÆEgyptiaca, Pers.

Eleusine Coracana, Pers.

Eleusine Indica, Gærtn., var. brachystachya, Trin.

Eleusine rigida, Spreng.

Leptochloa filiformis, Rœm. \& Schult.

Leptochloa mucronata, Kunth.

Leptochloa virgata, Beauv.

Koeleria phleoides, Pers.

Molinia cærulea, Mcench.

Eragrostis Brownei, Nees.

Briza minor, L. 


\section{.}


Glyceria festucæformis, Heynh.

Glyceria procumbens, Curtis.

Festuca ciliata, DC.

Festuca spectabilis, Jan.

Bromus arvensis, L.

Bromus pratensis, Lam.

Bromus multiflorus, Smith.

Bromus rubens, L.

Bromus squarrosus, L.

Lolium multiflorum, Lam.

Lolium rigidum, Gaud.

Lolium temulentum, L.

Lepturus incurvatus, Trin.

Hordeum murinum, L.

EQUISETACEAE.

Equisetum variegatum, Schlecht. 
. 







SMITHSONIAN INSTITUTION LIBRARIES 39088 00275761 5

\section{nhbohc QK117.T69}

Preliminary catalog of Anthophyta and Pt 